\title{
ESTUDIO DE LA PROGRESIÓN EN LA DELIMITACIÓN DE LAS «IDEAS» DEL ALUMNO SOBRE FUERZA
}

\author{
JIMÉNEZ GÓMEZ, E. ${ }^{1}$, SOLANO, I. ${ }^{1}$ y MARÍN, N. ${ }^{2}$ \\ 1 Departamento de Didáctica de las Ciencias Experimentales. Universidad de Murcia. \\ 2 Departamento de Didáctica. Universidad de Almería.
}

\section{SUMMARY}

The aim of this paper is to analyse if there has been any progress in the results that different papers about learners' «ideas» have shown in Dynamics. To that end, a wide sample of papers about «pupiIs' knowledge» concerning the concept of force has been chosen and a set of categories has been establihed in order to set up the criteria that allow us to compare the information presented in those papers.

\section{INTRODUCCIÓN}

El constructivismo, como método de enseñar ciencia, fundamenta su estrategia didáctica en el supuesto de que el alumno adquiera los contenidos objeto de enseñanza mediante una construcción activa a partir de "lo que sabe». No obstante, existen diversos modos de entender, averiguar y conceptualizar lo que entienden los investigadores y educadores por estos términos (Gómez et al., 1992).

Bajo «lo que el alumno sabe» subyacen términos, metodologías y marcos teóricos diferentes (Hashweh, 1988). Esta situación fue calificada hace ya más de diez años por Gilbert y Swift (1985) de fase «preparadigmática», por analogía con la descripción de Kuhn (1981) de los cambios científicos. En efecto, en esta fase, las distintas propuestas sobre «lo que el alumno sabe» han estado poco coordinađas, así: la metodología de investigación no es compartida, los objetivos últimos no están defini- dos, las implicaciones en el aula tampoco han sido coordinadas y la terminología no ha sido consensuada (Jiménez Gómez, Solano y Marín, 1994).

Un análisis lakatosiano realizado por Gilbert y Swift (1985), cornparando las aportaciones de la teoría de Piaget con las realizadas por el «movimiento de las concepciones alternativas» (MCA), llega a concluir que la teoría piagetiana estaba en periodo regresivo, mientras que el MCA presentaba indicios de cierta progresión.

Según Lakatos (1974 y 1983), el principal criterio para determinar si una teoría es progresiva es a través de su capacidad cle predecir hechos nuevos; es decir, su desarrollo teórico anticipa su desarrollo empírico. Este criterio sólo es válido si se utiliza para comparar programas de investigación en competencia, razón por la cual 
Gilbert y Swift lo aplican con las dos tendencias mencionadas. Sin embargo, Marín y Benarroch (1994) mostraron, comparando las aportaciones empíricas sobre concepciones en mecánica y naturaleza corpuscular de la materia, que no está claro que las aportaciones deI MCA superen las piagetianas, lo que pondría en entredicho la supuesta progresividad de1 MCA.

Siguiendo esta línea, en este trabajo se va a utilizar sólo uno de los requisitos lakatosianos de progresión, que se puede considerar como el menos exigente: «la teoría debe aportar nuevos datos empíricos de manera progresiva». De aquí, que se comparen las aportaciones empíricas de los distintos trabajos que han abordado el problema de delimitar las «ideas» que poseen los alumnos sobre el concepto de fuerza, con el fin de analizar si ha habido progresión en dicha delimitación. Así, si se ha producido una cierta progresión en el entramado teórico đel MCA, se debería apreciar también en los datos que aportan los distintos trabajos realizados sobre «ideas» de los alumnos a to largo de los años.

\section{SELECCIÓN DE TRABAJOS PUBLICADOS}

El principal objetivo de este trabajo consiste en mostrar si las publicaciones más recientes sobre «ideas» de los alumnos en dinámica aportan nuevos datos no contenidos en otrus realizadas con anterioridad.

Para conseguir el anterior objetivo se han llevado a cabo los siguientes pasos:

a) Selección de un contenido para el que se hayan realizado un buen número de trabajos sobre «ideas» espontáneas de los alumnos.

b) Elección de una muestra de trabajos representativos de los periodos a cubrir (antes y después de 1985, fecha de publicación de los trabajos de Gilbert y Swift), que permitan poner de manifiesto si ha existido progresión. en la delimitación de las «ideas» de los alumnos.

c) Establecer criterios que permitan comparar los datos aportados por los diferentes trabajos publicados.

\section{Selección de un contenido}

Con el fin de acotar el elevado número de trabajos realizados sobre las «ideas» que tienen los alumnos sobre ciencias (Carmichael et aI., 1990; Confrey, 1990), se optó por elegir la mecánica, por ser la fenomenología donde los alumnos han desarrollado el mayor número de esquemas espontáneos y donde más trabajos se han publicado.

El número de trabajos realizados en mecánica resultó ser excesivo, por lo que se seleccionaron sólo aquellos artículos que han abordado principalmente nociones ligadas al concepto de fuerza. De esta manera, la fuerza y conceptos con ella relacionados constituyen el conte- nido académico objeto de la investigación, pues sobre dicho contenido existe un número de trabajos publicados con anterioridad y posteridad al 1985 suficientes para cubrir las pretensiones del trabajo.

\section{Elección de una muestra de trabajos representativos de los periodos a cubrir}

Otro de los problemas es la selección de los trabajos que fuesen representativos de la investigación realizada sobre «ideas" de los alumnos relacionadas con el concepto de fuerza y que además permitiesen realizar el estudio de progresión en la delimitación «de lo que el alumno sabe» (Jiménez Gómez, Solano y Marín, 1994). Una dificultad añađiđa es que la mayoría de los trabajos están publicados en actas de congresos, reuniones o encuentros que son difíciles de obtener. De aquí, que se optara por seleccionar sólo aquéllos que hubiesen sido publicados en revistas de ámbito exclusivo de la enseñanza de las ciencias. No obstante, el número de revistas es elevado, por lo que se seleccionaron las siguientes, ordenadas alfabéticamente, que resultaron accesibles para nosotros y porque pensamos que cubren el objetivo de este trabajo:

1. Enseñanza de las Ciencias (1983-1985. El primer número apareció en 1983).

2. European Journal of Science Education, hoy International Journal of Science Education (1979-1995. EI primer número que se ha conseguido es del año 1979).

\section{Physics Education (1975-1995).}

\section{Science Education (1975-1995)}

Una vez seleccionadas las revistas, se ha fijado el periodo de revisión de publicaciones desde el 1979, año en que aparece el trabajo de Viennot, hasta el momento actual.

Para solucionar el problema de fijar un año para establecer los periodos a comparar se ha creído oportuno elegir el año 1985. Por un lado, porque es el año en que se publica el trabajo de Gilbert y Swift y, por otro, porque se pudo apreciar que existe una distribución análoga de trabajos en el periodo anterior y posterior al año seleccionado. La clasificación de los trabajos en los dos periodos, antes y después de 1985 , permite hacer comparaciones entre ambos grupos y observar si se ha producido progresión en la delimitación de las «ideas» de los alumnos, si bien, como se puede apreciar en las tablas I, III, IV y V, la mayoría de las comparaciones se han establecido en un continuo temporal, ordenando los trabajos por el año de publicación.

En total se han seleccionado veintinueve artículos, once que corresponden al periodo $1979-85$ y dieciocho at 1985-95. Pensamos que son los trabajos más significativos, aunque pudiera faltar alguno debido a que han utilizado palabras claves que no evoquen bien su conte- 
nido o porque su temática, aunque tenga cierta relación con el concepto de fuerza, no se han considerado de interés. Se han excluido también aquellas publicaciones de índole exclusivamente teórica, aunque hiciesen en algún momento referencia a «ideas» de los alumnos sobre el concepto de fuerza.

Los artículos seleccionados, ordenados por orden cronológico de publicación, aparecen en la tabla $I$, donde también se exponen las pretensiones de cada uno de ellos, la muestra utilizada y la técnica de recogida de datos.

\section{Criterios de comparación}

Observando la tabla I se puede apreciar la diversidad de pretensiones u objetivos de los diferentes trabajos, muestras y técnicas de recogida de datos (se presentan unas doce maneras diferentes de obtener datos). Esta heterogeneidad dejó entrever la dificultad de realizar estudios comparativos y, sobre todo, la necesidad de hacer agrupamientos en función de semejanzas y diferencias del tipo de aportaciones sobre «lo que el alumno sabe».

Del análisis de la tabla $I$, del tratamiento que los diferentes autores hacen de los datos (por falta de espacio no se presentan) y de los resuitados que ofrecen (algunos de los cuales se exponen en las tablas III, IV y V), se deduce que para unos «lo que el alumno sabe» acerca del concepto de fuerza son las explicaciones que realizan los alumnos sobre un determinado hecho físico, mientras que para otros son explicaciones o razonamientos independientes de los hechos físicos presentados. Por último cabría indicar que otros autores tienen en cuenta las explicaciones o razonamientos dependientes o independientes de los hechos físicos y además, por provenir dichas explicaciones o razonamientos de la estructura cognoscitiva del sujeto, establecen relaciones con variables de tipo cognitivo.

Lo anterior ha permitido establecer tres tipos de categorías que responden, en una primera aproximación, al modo en que los diferentes educadores o investigadores seleccionados conceptualizan las investigaciones referidas a «lo que el alumno sabe» sobre el concepto de fuerza. Así, se han establecido tres categorías, según quue los trabajos:

1. Identificuen y describan las respuestas de los alumnos y las agrupen por semejanzas o diferencias entre ellas, expresando los resultados mediante porcentajes de alumnos que han respondido de una manera u otra. Los resultados y conclusiones están ligados a las particularidades de los hechos físicos presentados en el cuestionario; a este grupo de trabajos, los hemos denominado descriptivos dependientes de los hechos físicos, y con estos términos se hará referencia más adelante a esta categoría.

2. Después de identificar y describir las respuestas de los alumnos, establezcan relaciones entre dichas respuestas referentes a las distintas preguntas realizadas y a los hechos físicos presentados. El grado de generatidad de las conclusiones a las que se llegan hace que los resultados que se ofrecen no estén ligados a dichos bechos e, incluso, algunos autores pueden predecir posibles respuestas de los alumnos a situaciones no presentadas en el cuestionario. La abstracción de los datos se hace desde la lógica del contenido objeto de búsqueda; en nuestro caso, Ios relacionados con el concepto de fuerza, su evolución histórica y con los conceptos con ella relacionados. Nos referiremos a los trabajos que están dentro de esta categoría con los términos descriptivos independientes de los hechos físicos.

3. Relacionen las respuestas obtenidas por los alumnos con otros factores o aspectos cognitivos que han mostrado ser relevantes, como el nivel cognoscitivo, el estilo cognitivo, la memoria a corto y largo plazo, las operaciones mentalts, etc. A estos trabajos les denominaremos descriptivos relacionales, en la medida que no establecen sus relaciones con otros factores cognoscitivos utilizando el contexto teórico en los que fueron estabiccidos dichos factores, sino tan sólo utilizando técnicas de estadística descriptiva.

En la tabla II se presentan las tres categorías establecidas en las que s:e pueden distribuir todos los trabajos seleccionados sobre dinámica. Las categorías y la clasificación de los diferentes trabajos se han realizado a partir del análisis de las pretensiones u objetivos, tratamiento de los datos y resultados ofrecidos en cada una de las publicaciones seleccionadas.

Esta categorización permite comparar datos entre los diferentes autores que están clasificados dentro de cada una de ellas, pero la diversidad de situaciones físicas presentadas para obtener información del alumno da lugar a una gran variedad de resultados que dificulta taI comparación. Por ello, se ha tomado finalmente como referente para hacer dichas comparaciones las situaciones físicas ıtilizadas en los cuestionarios, que responden en su mayoría a:

\section{Equilibrio estático.}

2. Lanzamiento hacia arriba o caída de objetos.

3. Desplazamiento de objetos en un plano horizontal.

4. Interacciones en movimiento relativo.

\section{Colisiones.}

En este trabajo, por razones de espacio, se han elegido aquellos hechos que responden a las situaciones físicas de lanzamiento hacia arriba o caída de objetos, por ser utitizadas por la mayoría de los trabajos seleccionados ( 21 de los 29 seleccionados) y porque se pueden realizar, en dichas situaciones, las comparaciones más significativas.

Las comparaciones entre los trabajos descriptivos relacionales se han realizado entre todos ellos, independientemente de las situaciones físicas presentadas, pues es posible dizha comparación al ser escaso el número de trabajos clasificados dentro de esta categoría. 


\begin{tabular}{|c|c|c|c|}
\hline Autor $y$ año & Pretensiones u objetivos del trahajn & Muestra & Técnica de recogida de datos \\
\hline Viennot, 1979 & $\begin{array}{l}\text { Explorar y analizar el razonamiento espontáneo de } \\
\text { estudiantes sobre dinámica elemental. }\end{array}$ & $\begin{array}{l}\text { Varios cientos de estudiantes principalmente franceses, } \\
\text { ingleses y belgas. La descripción que realiza de la mues- } \\
\text { tra es poco explícita. }\end{array}$ & $\begin{array}{l}\text { Test de papel y lápiz con cuestiones abiertas } \\
\text { sobre situaciones físicas. }\end{array}$ \\
\hline Helm, 1980 & $\begin{array}{l}\text { Obtener información cuantitativa de cómo de exten- } \\
\text { didas estaban una serie de conceptos erróneos o } \\
\text { ideas falsas en física. }\end{array}$ & $\begin{array}{l}\text { El test original fue aplicado a estudiantes de física } \\
\text { universizanios de primer año en Sudáfrica. Después: a } \\
334 \text { estudiantes de ciencias físicas de niveles equivalen- } \\
\text { tes al 0-level y A-level de escuelas blancas, a } 126 \text { de } \\
\text { escuelas públicas del mismo nivel y a } 65 \text { profesores. }\end{array}$ & $\begin{array}{l}\text { Test de cuestiones de elección múltiple donde } \\
\text { entre Ias opciones de respuesta se encontraban } \\
\text { conceptos erróneos co-munes o sospechados. }\end{array}$ \\
\hline $\begin{array}{l}\text { Watts, y } \\
\text { Zylbersziajn, } \\
\text { 1981 }\end{array}$ & $\begin{array}{l}\text { Conocer: a) la comprensión de los alumnos acerca } \\
\text { de la teoría de Newton y en qué medida los profeso- } \\
\text { res eran conscientes de ello; } b \text { ) si los esquemas } \\
\text { alternativos descritos en las publicaciones erancom- } \\
\text { partidos por sus alumnos. }\end{array}$ & $\begin{array}{l}125 \text { alumnos de comprehensive schools y sus } 5 \\
\text { profesores. }\end{array}$ & $\begin{array}{l}\text { Cuestionario con formato de elección múltiple. } \\
\text { Al final de cada cuestión se dejaba un espacio } \\
\text { donde se pedía al alumno tncluir una razón } \\
\text { para su respuesta. }\end{array}$ \\
\hline $\begin{array}{l}\text { Gunstone y } \\
\text { White, } 1981\end{array}$ & $\begin{array}{l}\text { Evaluar el nivel de comprensión de los alumnos } \\
\text { acerca del concepto de gravedad y de los principios } \\
\text { de la mecánica. }\end{array}$ & Estudjantes de física de $1^{\circ}$ de universidad. & $\begin{array}{l}\text { Pruebas de papel y lápiz con demostraciones. } \\
\text { Se pedía al alumno que escribiesen sobre sus } \\
\text { predicciones, observaciones y que las } \\
\text { comparase. }\end{array}$ \\
\hline Watts, 1982 & $\begin{array}{l}\text { Describir los esquemas conceptuales altemativos de } \\
\text { los niños sobre gravedad. } \\
\text { Proporcionar a los profesores rápidas y úties formas } \\
\text { de penetrar en el pensamiento de los niños. }\end{array}$ & $\begin{array}{l}20 \text { alumnos de } 1^{\circ} \text { a } 6^{\circ} \text { de escuelas secundarias de Londres } \\
\text { entre } 12 \text { y } 17 \text { años. }\end{array}$ & $\begin{array}{l}\text { Entrevistas personales de cuarenta minutos } \\
\text { utili-zando tarjetascon dibujos de líneas simples } \\
\text { que representan diversas situaciones donde el } \\
\text { concepto de fuerza es posible o no aplicarlo. }\end{array}$ \\
\hline $\begin{array}{l}\text { Selman y otros, } \\
1982\end{array}$ & $\begin{array}{l}\text { Comprobar la siguiente hipótesis: Existe una estruc- } \\
\text { tura general paralela entre el razonamiento lógico- } \\
\text { matemático y la comprensión de conceptos de } \\
\text { ciencias, no siendo ninguno de los dos dominios un } \\
\text { prerrequisito para el desarrollo del otro. }\end{array}$ & $\begin{array}{l}\text { Fase constructiva: } 50 \text { niños de primaria y } 10 \text { de high } \\
\text { school. } \\
\text { Fase de validacion: } 105 \text { sujetos en edades comprendidas } \\
\text { entre } 3 \text { y } 9 \text { años. }\end{array}$ & Entrevista clínica con algunas demostraciones. \\
\hline Watts, 1983 & $\begin{array}{l}\text { Describir las concepciones de los más jovenes sobre } \\
\text { fuerza. }\end{array}$ & $\begin{array}{l}20 \text { alumnos entre } 11 \text { y } 18 \text { años del área urbana de Londres } \\
\text { (junior y advanced). }\end{array}$ & $\begin{array}{l}\text { Entrevistas sobre ejemplos (dibujos esque- } \\
\text { máticos). }\end{array}$ \\
\hline Maloney, 1984 & $\begin{array}{l}\text { Investigar el conocimiento estratégico de los sujetos } \\
\text { al determinar cómo los individuos se ocupan de } \\
\text { tareas específicas. }\end{array}$ & I 12 estudiantes de high schools. & $\begin{array}{l}\text { Tests de elección múltiple. Una vez com- } \\
\text { pletado el cuestionario, se les pedía escribir } \\
\text { una explicación. }\end{array}$ \\
\hline Ivowi, 1984 & $\begin{array}{l}\text { Estudiar la extensión en que se dan una serie de } \\
\text { errores conceptuales en física entre los estudiantes } \\
\text { de cscuela secundaria y entre sus profesores que } \\
\text { permitan sugerir posibles causas y soluciones. }\end{array}$ & $\begin{array}{l}258 \text { estudiantes de quinto curso ( } 15-17 \text { años) de ocho } \\
\text { escuelas secundarias de Nigeria. } 10 \text { profesores de física } \\
\text { de esas escuelas. }\end{array}$ & $\begin{array}{l}\text { Test de lápiz y papel con items de elección } \\
\text { múltiple (las opciones para cada cuestión } \\
\text { contenian algunos errores de concepto cono- } \\
\text { cidos o sospechados). }\end{array}$ \\
\hline
\end{tabular}

Estudiar la extensión en que se dan una serie de de escuela secundaria y entre sus profesores que $-$
258 estudiantes de quinto curso (15-17 años) de ocho escuelas secundarias de Nigeria. 10 profesores de física mno tocluir una razon Pruebas de papel y lápiz con demostraciones. Se pedia al alumno que escribiesen sobre sus predicciones, observaciones y que las

Entrevista clínica con algunas demostraciones. Entrevistas sobre ejemplos (dibujos esqueests de elección múltiple. Una vez com tetado el cuestionario, se les pedía escribir Test de Jápiz y papel con ítems de elección multiple (las opciones para cada cuestion contenian algunos errores de concepto cono- 

$\begin{aligned} & \text { Ruggiero y otros, } \\ & 1985\end{aligned}$
i $\begin{aligned} & \text { Encontrar «esquemas espontáneosı que muestren la } \\ & \text { relación entre peso, airc y gravedad. }\end{aligned}$ |

- - - - - 1 - - - - -

Terry y otros, | Averiguar qué comprenden los niños sobre fuerzas y cquilibrio estático. Buscar estrategias de enseñanza.

Utiliza tres muestras: a) 22 alurnnos en el áltimo año una escueia media italiana (12-13 años), b) 8 alumo año de los anteriores. c) 40 alumnos de la misma escuela.

Alumnos de comprehensive schools: $\mathrm{N}=57\left(3^{\circ}\right)$ : No habian recibido instrucción sobre la $1^{\mathrm{a}}$ ni la $3^{\mathrm{a}}$ ley de Newton. $N=46\left(4^{\circ}\right)$ : se les habia introdacido en la $l^{\mathrm{a}}$ y la

$\mathrm{N}=55\left(5^{\circ}\right)$ : habían recibido instruccion sobre las tres leyes de Newton.

Terry y Jones, Investigar algunas de las dificultades conceptuale Que encuentran los estudiantes con la tercera ley de Newton y examinar las implicaciones que tengan para llevar a un cambio en su comprensión global del concepto de fuerza

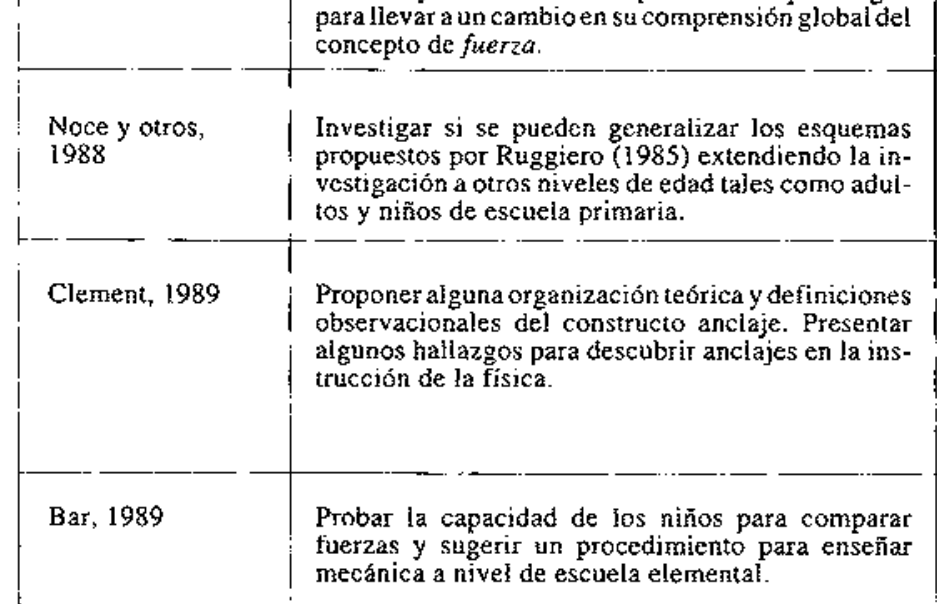

Brown, 1989

\begin{tabular}{l|l} 
creencia ingenua general de fuerza como una pro- \\
piedad innata o adquirida de los objetos. No \\
pretende obtener una lista de concepciones.
\end{tabular}

\begin{tabular}{l|l}
$\begin{array}{l}\text { Acevedo y otros, } \\
1989\end{array}$ & $\begin{array}{l}\text { Investigar las ideas previas de los alumnos cn diná- } \\
\text { mica, sclección de material de aprendizaje y evaluar }\end{array}$
\end{tabular}

\begin{tabular}{l|l}
$\begin{array}{l}\text { Acevedo y otros, } \\
1989\end{array}$ & $\begin{array}{l}\text { Investigar las ideas previas de los alumnos en diná- } \\
\text { mica, sclección de material de aprendizaje y evaluar }\end{array}$
\end{tabular} los resuitados de la enseñanza.
39 alumnos dc 16 años que recientemente habian completado un curso de física de nivel 0 .

a) 224 estudiantes de escuela secundaria, b) 64 estudiantes universitarios de primer año de biología, $c) 74$ adultos no expertos en física, d) 88 niños de $5^{\circ}$ carso de escuela primaria, e) 10 niños entre 8-12 años.

137 estudiantes de high school de 14, 15 y 17 años.

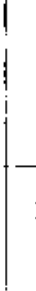

120 alumnos de escuela primaria entre $6 \cdot 11$ años a) 5 estudiantes de high school que no habian estudiado
física, b) 21 estudiantes de high school, c) 78 estudiantes de high school procedentes de siete clases de física. 65 estudiantes de $2^{\circ}$ de BUP (entre 15
y 18 años).
Cuestiones abiertas de lápiz y papel que se proponian oralmente después de una demostracion o que iban acompañadas de un dibujo aclaratorio.

Pruebas de lśpiz y papel donde se presentan diagramas.

Presenta a los alumnos dibujos que representan situaciones físicas y les pide interpretar las en términos de la tercerá ley de Newton.

Cuestionario de lápiz y papel con pregurtas abiertas. En el caso de la muestra $e$, utiliza además cntrevista clínica en la que se usan fotografías.

Test con 14 cuestiones de elección múltiple en cada una de las cuales también se pedia al alumno que indicara el grado de confianza en su respuesta mediante un numero en una escala que va del cero (suposición ciega) a

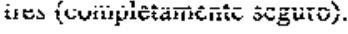

Test individual oral que consta de doce problemas de demostración.

a) Entrevistas orales de preguntas abiertas. b) Entrevistas con materiales escritos. c) Test de eleccion múltiple con seis problemas antes $y$ después de la instrucción.

Fase exploratoria: prueba abierta donde los alumnos tienen que completar un dibujo y da una explicacion, primero individualmente $y$ después en pequeños grupos. Fase de evalua ción: prueba individual similar a la inicial. 


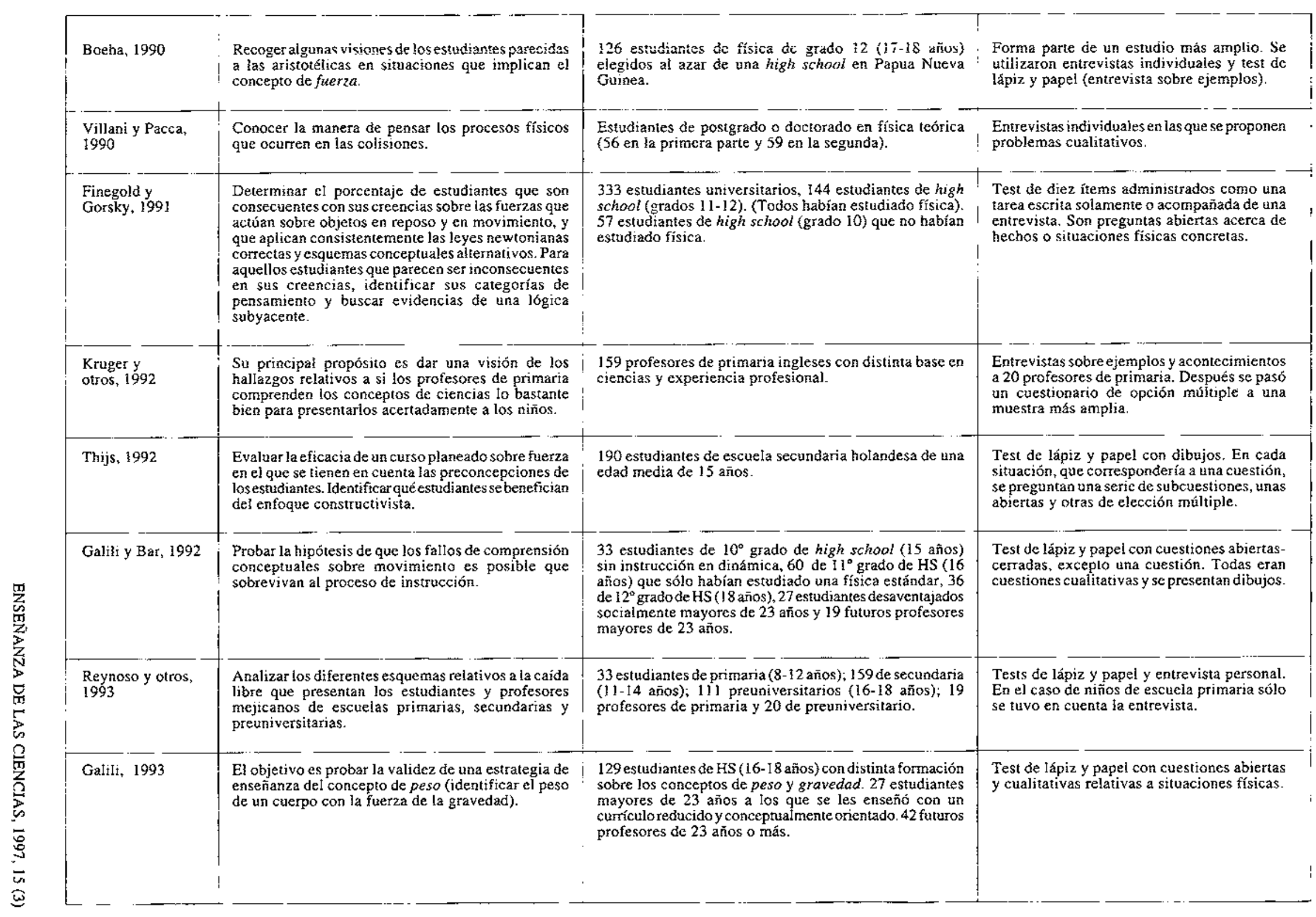




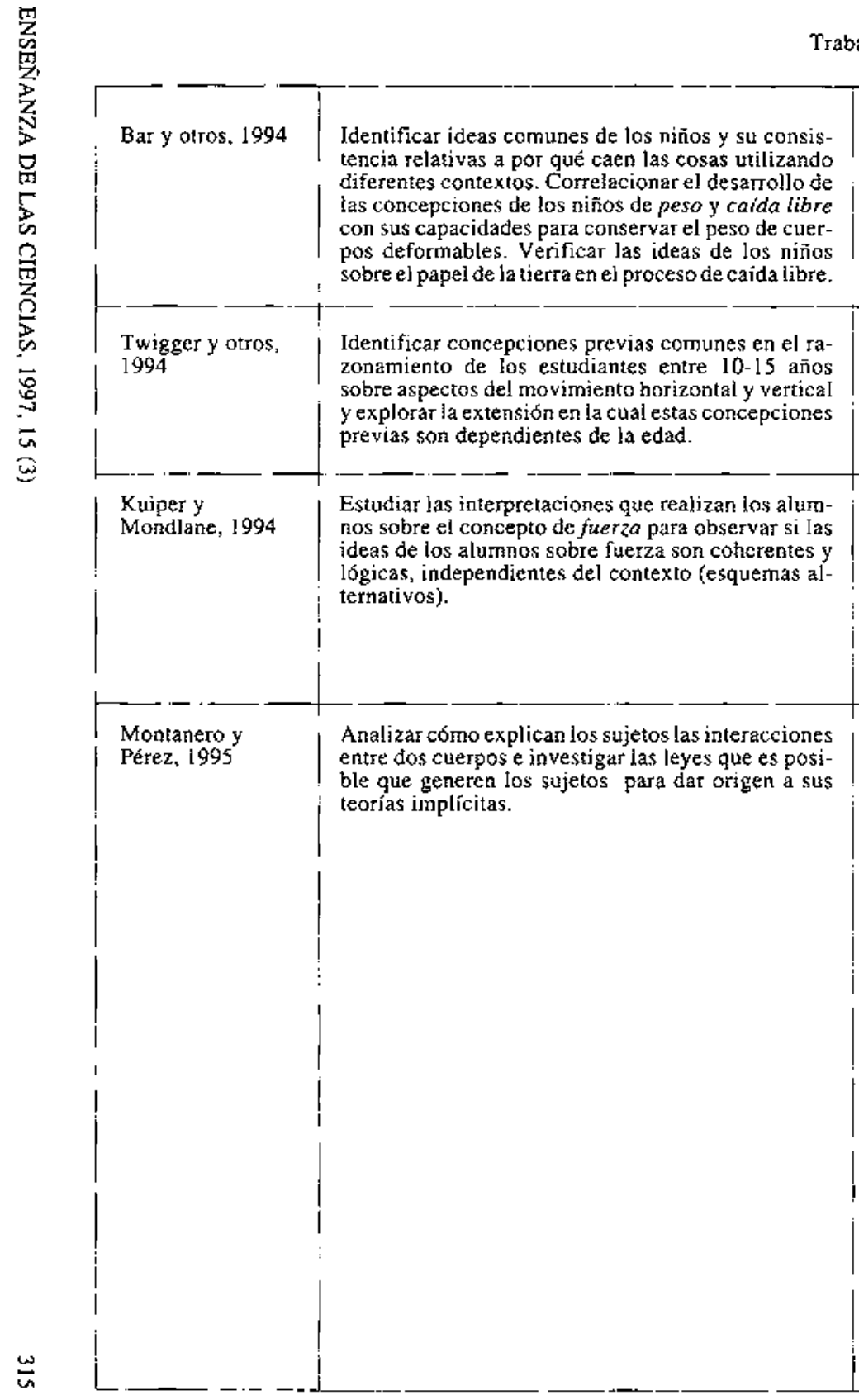

Tabla I

Trabajos seleccionados. (Continuación),

400 nin̆os de entre 4 y 13 años procedentes de una guardería, una escuela elemental y una junior high school. 20 chicos y 20 chicas fueron elegidos al azar de una poblacion total para cada nivel de edad.

Estos niñ no habín recibido enseñanza formal sobre peso y caída libre.

\section{6 estudiantes (10.15 años)}

Muestra principal: 143 sujetos (cursos desde $1^{\circ}$ a $6^{\circ}$ de secundaria) de Zimbabwe.

Estudio preliminar: 40 alumnos de 16-17 años. Estudio definitivo: 78 estudiantes de $7^{\circ}$ de EGB (12-13 años), 78

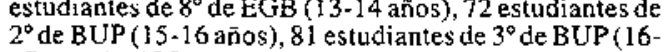
17 años), 105 estudiantes de COU (17-18 años), 47 estudiantes de $1^{\circ}$ de química $(18-19$ años), 21 estudiantes

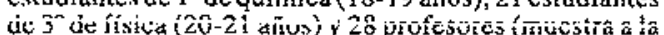
que se pasa el cuestionario)
Entrevista individual de media hora compuesta por cuatro tareas y presentada con ron las mismas tareas para todos jos grupos de edad.

Entrevistas de una hora realizadas en parejas de alumnos para fomentar la discusion. A cada estudiante se le pedia escribir o dibujar su respuesta.

Prueba mixta de papel y lápiz (opción múltiple y preguntas abiertas). En todos los casos se pide seleccionar una respuesta de un conjunto de items sobre la existencia de fuerzas y el nivel de certeza de su eleccion. Si la respuesta es alrmativa, se pidc dibujarlas, puesta es neyativa, se pide explicar por qué.

Estudio preliminar: test de lápiz y papel con opciones múltiples (se pide explicar la opcion escogida), entrevistas orales y discusiones de grupo. Estudio definitivo: nuevo test de lapiz y papel con opciones múltiples (se pidc explicar la opcion escogida), y un nume-

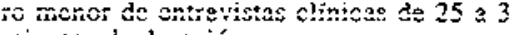
minutos de duración. 
Tabla II

Distribución por categorías de los trabajos seleccionados.

\begin{tabular}{|c|c|c|c|}
\hline Ā̃o & Descriptivos dependientes & Descriptivos independientes & Descriptivos relacionales \\
\hline$<85$ & $\begin{array}{l}\text { Helm, 1980; Gunstone y White, } \\
\text { 1981; Ivowi, } 1984\end{array}$ & $\begin{array}{l}\text { Viennot, 1979; Watts y } \\
\text { Zylbersztajn, 1981; Watts, } \\
\text { 1982; Watts, 1983; Maloney, } \\
\text { 1984; Ruggieroet al., 1985; } \\
\text { Terry et al., 1985 }\end{array}$ & Selman et al., 1982 \\
\hline$>85$ & $\begin{array}{l}\text { Terry y Jones, 1986; } \\
\text { Clement et al., 1989; } \\
\text { Brown, 1989; } \\
\text { Kruger et al., 1992; } \\
\text { Galili, } 1993\end{array}$ & $\begin{array}{l}\text { Noce et al., 1988; Boeha, } \\
\text { 1990; Villani y Pacca, 1990; } \\
\text { Finegold y Gorsky, 1991; } \\
\text { Thijs, 1992; Galili y Bar, } \\
\text { 1992; Reynoso et al., 1993; } \\
\text { Bar et al., 1994; Twigger et } \\
\text { al., 1994; Kuiper y Mondlane, } \\
\text { 1994; Montanero y Pérez, } \\
\text { 1995 }\end{array}$ & $\begin{array}{l}\text { Bar, 1989; Acevedo et al., } \\
\text { 9989 }\end{array}$ \\
\hline
\end{tabular}

\section{ESTUDIO COMPARATIVO DE LOS TRA- BAJOS SELECCIONADOS SOBRE «IDEAS» DE LOS ALUMNOS ACERCA DE FUERZA}

Para poner de manifiesto si ha existido progresión en los estudios realizados acerca de «lo que el alumno sabe» sobre el concepto de fuerza, se va a realizar una comparación entre los resultados que ofrecen los diferentes trabajos seleccionados que se encuentran en una misma categoría y que utilizan hechos físicos clasificados dentro de la situación física de lanzamiento hacia arriba o caída de objetos. Siguiendo estos criterios se han construido las tablas III y IV, correspondientes a las categorías descriptivos dependientes $e$ independientes de los hechos fisicos, respectivamente.

En la tabla V se comparan los diferentes trabajos clasificados como descriptivos relacionales, pero los hechos físicos que utilizan no pertenecen a la misma situación física.

En las tablas III, IV y V se presentan: en la primera columna, los autores y año de publicación; en la segunda, los hechos físicos; en la tercera, las actividades a realizar por el alumno; y, en la cuarta, los resultados más significativos obtenidos por los diferentes autores.

\section{Comparaciones entre los resultados obtenidos en los estudios categorizados como descriptivos dependientes, referidos a situaciones de lanzamiento o caída de objetos (Tabla III)}

En la tabla III, se pueden comparar autores que presentan los mismos hechos físicos y preguntas (Helm, 1980; Ivowi, 1984); análogo hecho físico que los anteriores pero modificando las cuestiones o actividades a realizar por el alumno (Gunstone y White, 1981); y, por último, los que cambian los hechos físicos y las actividades sobre dichos hechos (Terry y Jones, 1986; Clement et al., 1989; Brown, 1989; Kruger et al., 1992; Galili, 1993). De dicha comparación, se observa que: a) Helm (1980) e Ivowi (1984) presentan los mismos resultados; b) Gunstone y White (1981), al modificar el tipo de preguntas, encuentran resultados distintos; y c) los demás autores, al cambiar los hechos físicos y las preguntas, describen resultados diferentes entre sí y con respecto a los anteriores.

Los progresos que se producen en la categoría descriptiva dependiente de los hechos, si se comparan los trabajos posteriores a 1985 con los anteriores, se reficren fundamentalmente a la presentación de nuevos resultados, como consecuencia de modificar aspectos perceptivos en las situaciones físicas que se presentan al alumno (caida libre de una piedra o de un ascensor), lo que origina respuestas diferentes, aunque se realicen análogas preguntas (Galili, 1993).

A pesar de que se ofrecen resuitados diferentes y puntualizaciones de carácter metodológico, como las que apunta Galili (1993), no parecen existir diferencias entre los resultados publicados por los diferentes investigadores en el periodo anterior a 1985 y el posterior, en cuanto que los distintos resultados que se presentan en esta categoría se deben, más que a una progresión en la metođologia o al marco teórico, a los hechos físicos utilizados, al tipo de pregunta realizada y a la muestra seleccionada.

Resulta ilustrativo que todos los autores, a excepción de Gunstone y White (1981), utilicen en sus preguntas el término fuerza. De aquí que las respuestas del alumno 


\begin{tabular}{|c|c|c|c|}
\hline Autores & Hechos fisicos & Actividad a realizar por el alumno & Resultados encontrados \\
\hline Helm, 1980 & $\begin{array}{l}\text { 1. Dos bloques }(A \text { y } B \text { ) unidos por una cuerda que pasa por } \\
\text { una polea, en uno de sus extremos esta } A \text { que cae vertical. } \\
\text { mente y } B \text { que se desliza por la mesa. }\end{array}$ & $\begin{array}{l}\text { 1. Identificar quién ejerce la fuerta que } \\
\text { actúa sobre } B \text {. }\end{array}$ & $\begin{array}{l}\text { En el trabajo se presentant los porcentajes para las respuestas correctas y de } \\
\text { algunos de los distractores más populares (conceptos emóneos o ideas } \\
\text { falsas). } \\
\text { 1. La fuerza } F \text { que actúa sobre } B \text { es ejercida por: a) bloque } A, b \text { ) la tierra, } \\
\text { c) la cuerda, d) la mesa. }\end{array}$ \\
\hline $\begin{array}{l}\text { Gunstone y White, } \\
1981\end{array}$ & 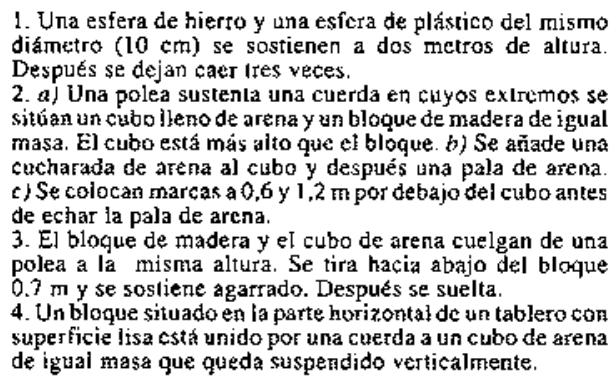 & $\begin{array}{l}\text { 1. Predecir la relación cntre los tiempos } \\
\text { que tardan en caer. Registrar las observa- } \\
\text { ciones y explicar posibles discrepancias. } \\
\text { 2.a) Predecircuálde los dos objelos pesa } \\
\text { más y explicar las bases para hacer la } \\
\text { predicción. } \\
\text { b) Predecir qué puede ocurrir con el } \\
\text { cubo, observar y comparar. } \\
\text { c) Predecir la relación entre las velocida- } \\
\text { des del cubo con las dos marcas, observar } \\
\text { y comparar. } \\
3 \text { y } 4 \text {. Predecir lo que ocurrirá cuando se } \\
\text { libere el bloque, observar y comparar. }\end{array}$ & $\begin{array}{l}\text { Niveles de comprensión (adecuados o inadecuados): } \\
\text { 1. Cuando la observación entra cn conflicto con la predicción, la respuesta } \\
\text { usual cs decir que la resistencia del aite cs mentor o mayor de lo que se } \\
\text { anticipa A continuación, la respuesta más comúnes ignorar la discrepancia. } \\
\text { 2. a) Pesan igual, el bloque pesa más o el cubo pesa más. b) La mayoría eran } \\
\text { correctas. c) Velocidad en la narca más baja, mayor que cn la más alta } \\
(90 \% \text { ). } \\
\text { 3. Crcencia de muchos estudiantes de que el sistema volvería a su posicicion } \\
\text { original. } \\
\text { 4. Cuanto más espectacular sea la observación y, por tanto, mayor la } \\
\text { discrepancia con la predicción, existe menos tendencia a justificar la } \\
\text { predicción y más tendencia a considerarla como errónea. }\end{array}$ \\
\hline Irowi, 1984 & $\begin{array}{l}\text { 1. Dos bloques }(A \text { y } B) \text { unidos por una cuerda que pasa por } \\
\text { una polea, en uno de sus extremos está } A \text {, que cae vertical- } \\
\text { mente, y } B \text {, que se desliza por la mesa } \\
2 \text {. Un cuetpo } B \text { esta situado encima de otro } A \text {, que a su vez } \\
\text { está en el borde de una mesa. Se da un fuerte empunon a } A \text {, } \\
\text { de tal manera que } A \text { sale lanzado con un componente } \\
\text { horizontal de ia velocidad y } B \text { cac verticalmente hacia } \\
\text { abajo. }\end{array}$ & $\begin{array}{l}\text { 1. Identificar quién ejerce la fuerza que } \\
\text { actúa sobre } B \text {. } \\
\text { 2. Fredecir quéc cuerpo alcanza primero el } \\
\text { suelo y explicat por qué. }\end{array}$ & 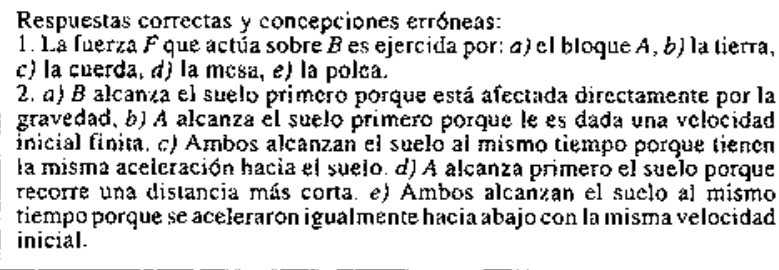 \\
\hline Terry y Jones, 1986 & Una piedra cae verticaimente hacia la tierra. & $\begin{array}{l}\text { ldentificar la fuerza que está emparejada } \\
\text { con la fuerza gravitacional de ia tierra. }\end{array}$ & $\begin{array}{l}\text { Respuestas cróneas o correctas: } \\
\text { a) Respuesta correcta ( } 4 \text { alumnos). } \\
\text { b) La resistencia del aire ( } 18 \text { aiumnos). c) No sugieren una fuerza ( } 17 \\
\text { alumnos). }\end{array}$ \\
\hline Galili, 1993 & Un ascensor coat una persona dentro en estado de caída libre. & $\begin{array}{l}\text { 1. Identificar las fuerzas experimentadas } \\
\text { por la persona y describir las sensaciones } \\
\text { que tiene la persona antes del impacto. }\end{array}$ & 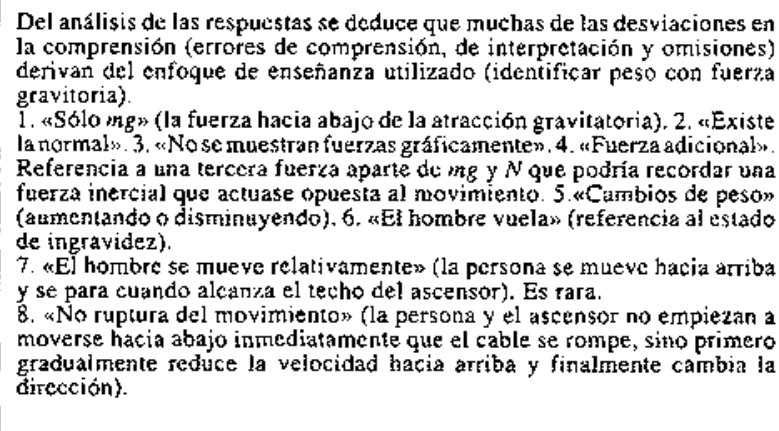 \\
\hline
\end{tabular}


Tabla IV

Trabajos descriptivos independientes de los hechos físicos prescntados.

\begin{tabular}{|c|c|c|c|}
\hline Autores & Hecilus ftsicus & Actividad a realizar por el alumno & Resultados encontrados \\
\hline $\begin{array}{l}\text { Viennott, } \\
1979\end{array}$ & $\begin{array}{l}\text { Movimientocongeladoenuninstante } \\
\text { determinado y a la misma altura } \\
\text { de: I) Una serie de pelotas de } \\
\text { majabarista que han sido lanzadas } \\
\text { hacia arriba siguiendo dos } \\
\text { trayectorias distintas y que varian } \\
\text { en cuanto a las características del } \\
\text { vector velocidad. (Resistencia del } \\
\text { aire despreciable). 2) Tres masas } \\
\text { idénticas suspendidas de muelles } \\
\text { colgados del techo que tienen } \\
\text { movimiento periodico de distinta } \\
\text { amplitud pero que en ese instante } \\
\text { presentan la misma elongacion y } \\
\text { distintos vectores velocidad. }\end{array}$ & $\begin{array}{l}1 \text { y } 2 \text {. Comparar las fuerzas que } \\
\text { actúan sobre cada una de las masas. }\end{array}$ & $\begin{array}{l}\text { Tendencias generales en el razonamiento espontáneo: } a \text { ) Tendencia a atribuir } \\
\text { cantidades físicas (p.e., fuerza o energía) a los objetos mismos. } b \text { ) Tendencia a buscar } \\
\text { una fuerza (o sentir una necesidad para una fuerza) en la dirección del movimiento } \\
\text { para explicar el movimiento. } \\
\text { Ley intuitiva sobre movimiento: una relación pseudotineal entre fuerza y velocidad, } \\
F \text { a } \alpha(v) \text {. } \\
\text { 1. Si } \nu=0 \text {, entonces } F=0 \text {, incluso si la aceleracion no es cero. } \\
\text { 2. Si } \nu \neq 0 \text {, entonces } F \neq 0 \text { incluso si } a=0 \text {. } \\
\text { 3. Si las velocidades son diferentes, las fuerzas son diferentes, incluso si las } \\
\text { aceleraciones son las mismas. } \\
\text { Nociones de fuerza: } \\
\text { a) Fuerza de interacción ( } F a_{\text {a }} \text { ) es función de la posición del cuerpo que se mueve, y } \\
\text { determina el rango de canbio de velocidad; } F a c=\text { ma. Es escogida para explicar el } \\
\text { movimiento cuando éste no es conocido como informacion inicial y en un sistema } \\
\text { galileano cuando la fuerza actúa en la misma direccion del movimiento. } \\
\text { b) Fuente de fuerza }(F \text { ) considerada como ola fuerza en un cuerpo que lo mantiene } \\
\text { en movimienton. }\end{array}$ \\
\hline $\begin{array}{l}\text { Watts y } \\
\text { Zylbersztajn. } \\
1981\end{array}$ & $\begin{array}{l}\text { 1. Una piedra lanzada verticalmente } \\
\text { hacia arriba. } \\
\text { 2. Una bala disparada por un cañón } \\
\text { gue sigue una trayectoria parabólica. } \\
\text { 3. Un astronauta sobre la luna deja } \\
\text { caer una llave inglesa. }\end{array}$ & $\begin{array}{l}\text { 1 y 2. Identificar las fuerzas que } \\
\text { actúan sobre la piedra o la bala en } \\
\text { varios puntos de su trayectoria. } \\
\text { 3) Predecir lo que ocurrirá con la } \\
\text { llave inglesa. } \\
\text { En todos los casos se pedía dar una } \\
\text { explicación. }\end{array}$ & $\begin{array}{l}\text { 1 y 2. Esquemas alternativos: Si un cuerpo está moviéndose, existe una fuerza neta } \\
\text { sobrc él en la dirección del movimiento. Si el cuerpo no se estă moviendo, no existe } \\
\text { fuerza neta actuando sobre él. } \\
\text { 3. Opciones mayoritarias: } \\
\text { - La llave inglesa permanecería estacionaria a la altura de la mano sobre la superficie } \\
\text { de la luna. } \\
\text { - Ascendería hacia arriba lejos de la superficie. } \\
\text { (Juntas corresponderían al } 80 \% \text { ). }\end{array}$ \\
\hline Watts, 1982 & $\begin{array}{l}\text { 1. Un jugador de golf golpeando } \\
\text { una pelota de golf. } \\
\text { 2. Un astronauta que tropieza con } \\
\text { un cráter en la luna. } \\
\text { 3. Un bañista saltando sobre un } \\
\text { trampolín. }\end{array}$ & $\begin{array}{l}\text { Identificar si existen o no fuerzas } \\
\text { en cada situación. }\end{array}$ & $\begin{array}{l}\text { Esquemas alternativos: } \\
\text { - La gravedad es una fuerza que requiere un medio a través del cual actuat. } \\
\text { - Donde no existe aire, no existe gravedad. } \\
\text { - La gravedad se incrementa con la altura. } \\
\text { - La gravedad es constante -los objetos que se mueven intentan contramestar la } \\
\text { gravedad y fallan. } \\
\text { - La gravedad empieza a operar cuando los objetos empiezan a caer y continúa hasta } \\
\text { que están en reposo sobre el suelo. } \\
\text { - La gravedad es una fuerza grande. } \\
\text { - La gravedad es grande porque actúa sobre muchos objetos. } \\
\text { - La gravedad es selectiva -no actúa sobre todas las cosas de la misma manera ni } \\
\text { sobre las mismas cosas todo el tiempo. } \\
\text { - Gravedad no es peso -pero puede actuar en conjuncion con el peso para mantener } \\
\text { en su sitio las cosas. }\end{array}$ \\
\hline Watts, 1983 & $\begin{array}{l}\text { 1. Un golfista golpeando la pelota. } \\
\text { 2. Un astronauta que tropieza } \\
\text { con un cráter en la Luna. } \\
\text { 3. Un niño saltando a la piscina } \\
\text { desde un trampolín. }\end{array}$ & $\begin{array}{l}\text { Identificar la presencia o ausencia } \\
\text { de fuerzas en cada situación. Dar } \\
\text { una explicación. }\end{array}$ & $\begin{array}{l}\text { Esquemas alternativos: } \\
\text { - Fuerzas afectivas (que conducen a una acción): fuerzas son obligaciones para } \\
\text { realizar una accion en contra de alguna resistencia. } \\
\text { - Fuerzas de configuración: los objetos retenidos en una posición tienen fuerza. } \\
\text { Tienen un sentido similar a la idea de! físico de energia potencial. }\end{array}$ \\
\hline
\end{tabular}

1. Un jugador de golf golpeando
una pelota de golf. 2. Un astronauta que tropieza con un crater en la luna.

3.Un bañista saltando sobre un

1. Un golfista golpeando la pelota. 3. Un niño saitando a la piscina
Identificar la presencia o ausencia de fuerzas en cada situación. Da
1 y 2. Comparar las fuerzas que Ley intuitiva sobre movimien $F=\alpha(y)$ 3. $\mathrm{Si}$.

a) Fuerza de interacción ( $\mathrm{Fa}$ ) es función de la posición del cuerpo que se mueve, determina el rango de canbio de velocidad, $F a_{\alpha}=m a$. Es escogida para explicar el alileano cuando la fuerza actúa en la misma direccion del rnovimiento.

b) Fuente de fuerza $(F)$ considerada como xla fuerza en un cuerpo que lo mantiene

Esquemas altemativos: Si un cuerpo está moviéndose, existe una fuerza net fuerza neta actuando sobre é.

tallones mayoritarias. e la luna.

ría hacia arriba lejos de la superficie.

Esquemas alternativos:

entrastestar la

que están en reposo sobre el suelo.

- La gravedad es grande porque actía sobre muchos objetos.

es selectiva -no actua sobre todas las cosas Cobre las mismas cosas todo el tiempo.

Esquemas alternativos:

- Fuerzas de configuración: los objetos retenidos en una posición tienen fuerza.

Tienen yn sentido similar a la idea del físico de energia potencial. 
Fuerzas denominadas: son inherentes a aquellos objetos que hacen que ocura un suceso. Los humunos, las máquinas y algunos objetos son centros de fuerza: noción antropomórfica de fuerza.

- Fuerzas de encuentro: consideran $F$ como entidad única, más que como interacción entre cuerpos. Cuando dos o más fuerzas se juntan, ellas pueden combinarse para cambiar el movimiento de un objeto.

- Fuerza móvil: fuerza es algo que se requiere para causar y mantener el movimiento. - Fuerzas operativas: fuerza es una acción. La cantidad de fuerza es proporcional a a cantidad de actividad que tiene lugar; es similar a la noción del físico de energía - Fuerzas denominadas: son inherentes a aquellos objetos que hacen que ocurra un suceso. Los humanos, las máquinas y algunos objetos son centros de fuerza: noción ntropomórfica de fuerza.

- Fuerzas de encuentro: consideran $F$ como entidad única, más que como interacción entre cuerpos. Cuando dos o más fuerzas se juntan, ellas pueden combinarse para cambiar el movimiento de un objeto.

Fuerza móvil: fuerza es algo que se requiere para causar y mantener el movimiento Fuerzas operativas: fuerza es una acción. La cantidad de fuerza es proporcional a

Esquemas de conocimiento espontáneo:

Esquema A: La fuerza de la gravedad opera sobre el peso de los objetos causando su caida La presión del aire es la causa de ta gravedad Esquema Al: En ausencia

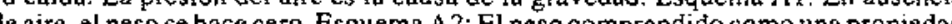
de los objetos no varía. Esquema B: La fuerza de la gravedad y el peso son causas independientes que dan lugar a la cáda de los objetos. Esquema B 1: Peso y gravedad dependen del aire. Esquema B2: El aire opera sólo sobre el peso o sólo sobre la gravedad.

Esquema B2. El aire opera solo sobre el peso solo sobre la gravedad. Eue de caida coscona de los fenómenos sobre la Tierra pero la gravedad es una propiedad sólo de la Tierra yel sacio delos denćlos

Esquema D: Corresponde al científicamente correcto.

* Muestras $a$, $b$ y c: Analiza las respuestas en base a los criterios de Ruggiero (1985) de esquemas de conocimiento espontáneo (esquemas A, B, C y D; Ruggiero, 1985). * Mucstra $d$ : Los criterios de Ruggiero no se pueden aplicar a toda la muestra. 1 y 2 . Respuestas posibles: flota (79), cae (8), no contesta (1). Se analizan las respuestas de 55 niños que habian dicho que la llave flota sobre la Luna y daban definiciones de fuerza de gravedad articuladas:

- Enfogue o modelo descriptivo. No explican por qué flota la llave y den una definición genérica de gravedad (pe es un tipo de aire es una fuerza que atrac) (21 niños). qué fló la llave $y$ al definir gravedad la rueciona con otras respuestas anteriores distingu con la Tierra y el que los objetos floten cerca de la superficie de la Luma es debido anda fuerza de gravedad originada desde la Tierra y extendida en el espacio y que 
Tabla IV

Trabajos descriptivos independientes de los hechos físicos presentados. (Continuación).

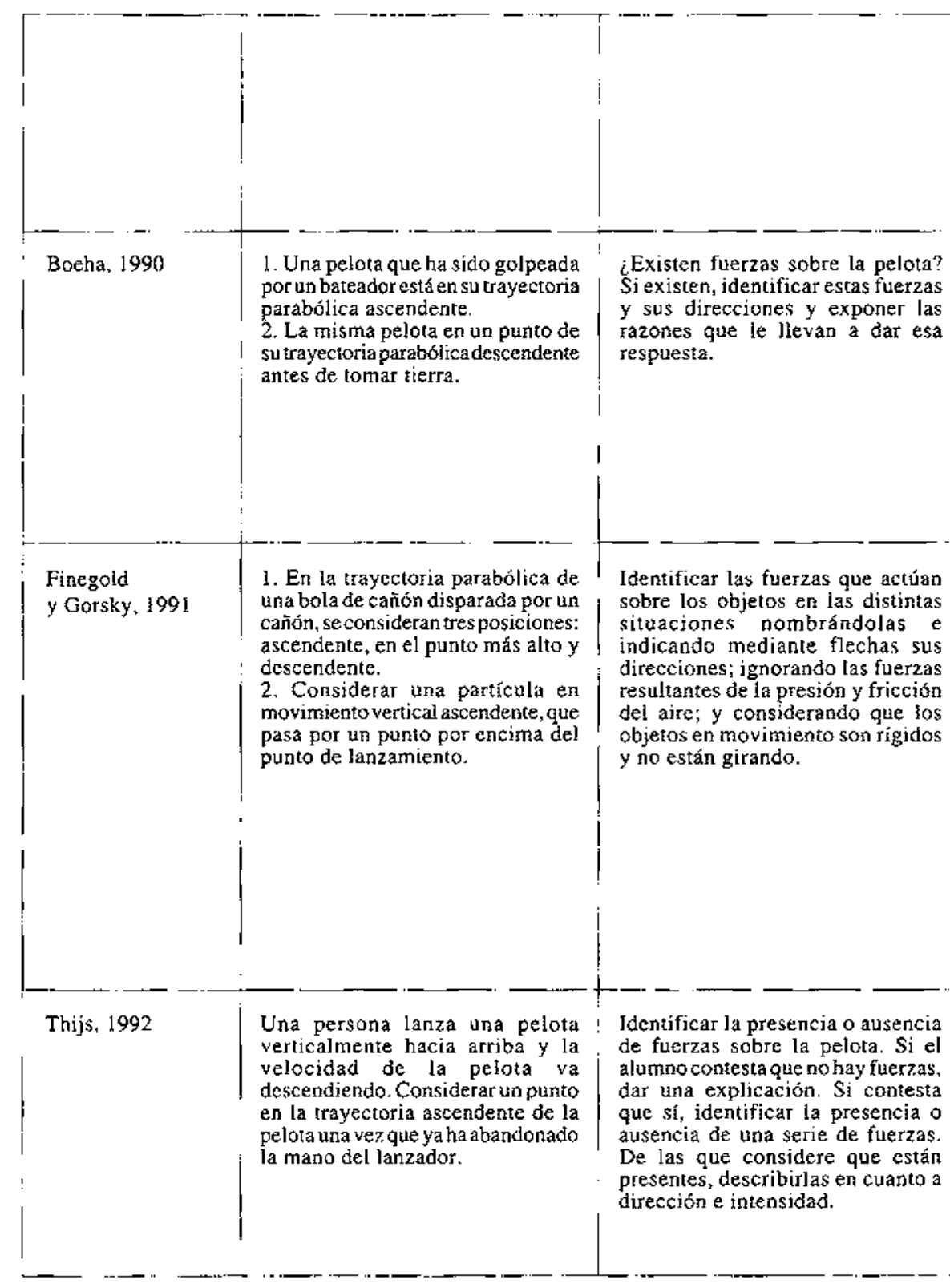

atrae las cosas hacia la Tierra) y definiciones tautológicas de gravedad ( $\alpha$ s la fuerza que hace que las cosas floten").

2. Esquemas de Ruggiero (1985): Esqucma A (11 alumnos), esquema B (6 alumnos), esquema $C(21)$

No responde (49 alumnos)

Muestra explicativo geocéntrico del hecho observado de flotar los objetos sobre la Luna.

Visiones de tipo aristotélico:

- Fuerzas denominadas: Ciertos cuerpos u objetos están dotados de fuerza. Poseen - Fuerzas denominadas: Ciertos cuerpos u objetos están dotados do

- Fuerzas operativas: Fuerza como una acción que puede producir un efecto. Se consume proporctonalmente al nivel dc actividad, desaparece o se transforma de una Fue en ruerzas de encuentro. Si as fuerzas se retnen, a mendo no producen movimiento y, y la resistencia del aire.

- Muchos estudiantes en la muestra, antes de la instrucción formal en física, se adhiercn a creencias cientificamente ineorrectas sobre las fucrzas que actúan sobe objetos en movimiento.

li esquema "novimiento implica una fucrza neta» no es consistentemente aplicado, inciuso por alumnos que no han estudiado física. Los datos sugieren que el esquema explicativo "movimiento implica fuerza..." señalada por Viennot (1979), Clement (1982, 1983) y Watts (1983) noes un buen predictor de las crecncias de los estudiantes relativas a objetos aresufen proyectiles. Los anteriores investigadores trabajaron con sistemas únicos y no con

- Se identifican categorias conceptuales aparentemente consistentes y coherentes en conjuntos de respuestas que actuan sobre objetos en movimiento. Esto es, los conjuntos de respucstas que actian - Probablemente, existen esquemas en la mente de los estudiantes como «depósitos. para la asignatura de fisica: sinembargo, exceptopara los estudiantes que consistentemente para la asignatura de fisica, sinembargo, exceptopara los estudiantes que consistentemente creencias de los estudiantes sobre comportamiento físico sea posible predecir.

Para elaborar el cucstionario utiliza términos extraídos de investigaciones de otros autores bajo los cuales subyace una concepción enónea particular. Estas concepciones erróneas son:

. Cuando un objeto se mueve, una fuerza inherente al objeto (ímpetu) expifica su movimiento.

La fricción tiene un valor constante y no tiene una dirección particular

. La fuerza conductora se asocia con el movimiento. La fuerza de la gravedad desciende cuando el balón sube. es cero en la parte alta de la trayectoria y se 4. La fura resultate

mis dira resultante tiene la misma dirección oute la velocidad del objeto y su valor cambia de la misma forma. 
Tabla IV

Trabajos descriplivos independientes de los hechos físicos presentados. (Continuación).

\begin{tabular}{|c|c|c|c|}
\hline $\begin{array}{l}\text { Galili y Bar, } \\
1992\end{array}$ & $\begin{array}{l}\text { 1. Un cuerpo que ha sido lanzado } \\
\text { hacia arriba. } \\
2 \text {. Un ascensor con un pasajero } \\
\text { dentro se está moviendo con velo- } \\
\text { cidad constante hacia arriba y ha- } \\
\text { cia abajo. }\end{array}$ & $\begin{array}{l}\text { 1. Identificar y nombrar lodas las } \\
\text { fuerzas ejercidas sobre el cuerpo } \\
\text { (omitjendo cl razonamiento). } \\
\text { 2. Identificar las fuerzas cjercidas } \\
\text { sobre el pasajero. }\end{array}$ & $\begin{array}{l}\text { 1. a) Respuesta correcta. b) Fucrza hacia delante. c) Fuerza cero en la parte superior } \\
\text { de la trayectoria. } d \text { ) Sin respucsta. } \\
\text { 2. a) Suma de fuerzas cero. b) Fuerzas identificadas correctamente. c) Uso del } \\
\text { principio de Galileo. } d \text { ) Hacia arriba no igual que hacia abajo. e) La tercera fuerza } \\
\text { (mencionar la fuerza del cable). f) fuerza m.a. g) Integración (afirmaban que las } \\
\text { fuerzas ejercidas sobre el ascensor eran transferidas y sentidas por la persona). } h \text { ) Sin } \\
\text { fuerza Normal. i) Sin mg. } \text { () No responden. } \\
\text { Algunas de las respuestas enoneas responden a visiones prenewtonianas ya descri- } \\
\text { tas anteriomente por otros autores, por ejemplo: «fuerza es proporcional a veloci- } \\
\text { dad» o «movimiento implica fuerza». }\end{array}$ \\
\hline & & & \\
\hline $\begin{array}{l}\text { Reynoso y otros, } \\
1993\end{array}$ & $\begin{array}{l}\text { 1. Un astronauta en la Luna lleva } \\
\text { una piedra cn la mano y la suelta. } \\
\text { 2. La misma situación pero en la } \\
\text { Tierra. } \\
\text { 3. Un astronauta sobre la Luna tie- } \\
\text { ne dos hojas de papel idénticas. } \\
\text { Una de ellas la estruja con la mano } \\
\text { formando una bola y la otra no la } \\
\text { toca. Después, las deja caer. } \\
\text { 4. La misma situación pero en la } \\
\text { Tierra. } \\
\text { 5. Levantar una piedra pesada en la } \\
\text { Luna y en la Tierra. }\end{array}$ & \begin{tabular}{|l} 
1 y 2. Describir qué ocurte y expli- \\
car por qué. \\
3 y 4 . Describir qué ocurte y expli- \\
i car por qué. Cuestiones diseñadas \\
para explorar las ideas de los suje- \\
tos sobre peso, densidad e influen- \\
cia del medio cn la caída de los \\
cuerpos. \\
5. Comparar la facilidad para le- \\
vantar una piedra pesada en la Tie- \\
ra y en la Luna. Cuestiones diseña- \\
das para descubrir las ideas sobre la \\
relaciơn entre peso y gravedad. \\
Las siguientes cuestiones se pre- \\
guntan sin hacer referencia a situa- \\
ción alguna. \\
6. Definir el peso de un cuerpo. \\
7. Definir qué es la gravedad.
\end{tabular} & 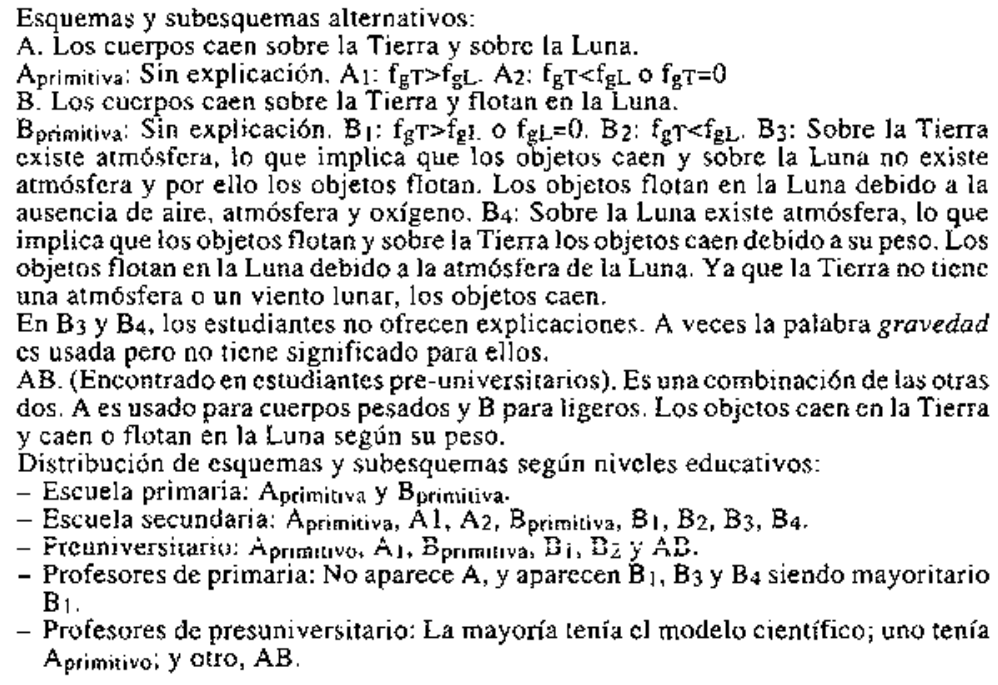 \\
\hline $\begin{array}{l}\text { Bar y otros, } \\
1994\end{array}$ & $\begin{array}{l}\text { 1. Un objeto que cae al suelo. } \\
\text { 2. Un trozo de hielo flota en el agua } \\
\text { y un trozo de plastilina se hunde. } \\
\text { 3. Los barcos flotan en el agua. } \\
\text { 4. Dos cubos de las mismas dimen- } \\
\text { siones, uno más pesado que el otro, } \\
\text { se dejan caer al mismo tiempo des- } \\
\text { de la misma altura. }\end{array}$ & $\begin{array}{l}\text { 1. Explicar por qué cae el objeto y } \\
\text { por qué lo hace hacia abajo. } \\
\text { 2. Explicar por qué el hielo flota y } \\
\text { la plastitina se hunde. } \\
\text { 3. Explicar por qué los barcos flo- } \\
\text { tan en el agua. } \\
\text { 4. Comparar los pesos sosteniéndo. } \\
\text { los en la mano. Predecir qué peso } \\
\text { llegará antes al suelo. }\end{array}$ & $\begin{array}{l}\text { 1. Idcas de los niños de por qué las cosas caen. Tres categorías: a) ausencia de soporte, } \\
\text { b) pesadez, c) la fuerza atractiva de la tierra. } \\
\text { 2. Predicciones sobre los objetos que caen. Tres categorias: a) El objeto ligero } \\
\text { precede al objeto pesado ( } 5-7 \text { años), b) El objeto pesado llega antes ( } 7 \text { - años). c) El } \\
\text { objeto ligero y el pesado alcanzarán la tierra juntos ( } 13 \text { años, respuesta correcta } \\
\text { aunque explicación incorrecta). } \\
\text { 3. Conservación del peso de objetos deformables. La conservación del peso aumenta } \\
\text { con la edad. } \\
\text { 4. Relación entre peso y caída libre. Las ideas de los niños pueden ser interpretadas } \\
\text { en términos de una teorfa de sentido común significativa. } \\
\text { (5-7 años): Caer es un movimiento natural y para impedir que algo caiga se necesita } \\
\text { esfucrzo (suele implicar conexión tangibic). } \\
\text { Identifican peso con fuerza -la fuerza de choque, la fuerza para penetrar, la fuerza } \\
\text { presionando sobre la mano de uno y la fuerza interna- que puede mantencr algo } \\
\text { estable. }\end{array}$ \\
\hline
\end{tabular}

1. Un cuerpo que ha sido lanzado 1. Lentificar y nombrar lodas las
hacia arriba. 2. Un ascensor con un pasajcro fomitiendo cl razonamiento) Una piedra cn la mano y la suelta.

3. Un astronauta sobre la Luna tiene dos hojas de papel identicas. forme des la estruja con la man oca. Después, las deja caer.

Tierra.

S.Levantar una piedra pesada en la Luna y en la Tierra.

guntan sin has coestiones se precion alguna.

6. Definir el peso de un cuerpo.$$
\text { . }
$$

- Profesores de primaria: No aparece A, y aparecen $B_{1}$ B $B_{3}$ y $B_{4}$ siendo mayoritario B

1. Idcas de los niños de por qué las cosas caen. Tres categorías: a) ausencia de soporte,

predicciones sobre los objetos que cacn. Tres categorias: a) El objeto ligero objeto ligero y el pesado alcanzarán la ticrra juntos (13 años, respuesta correct

4. Relación entre peso y caída libre. Las ideas de los niños pueden ser interpretada

esfuerzo (suele implicar conexión tangible). 
Tabla IV

Trabajos đescriptivos independientes de los hechos físicos presentados. (Continuación).

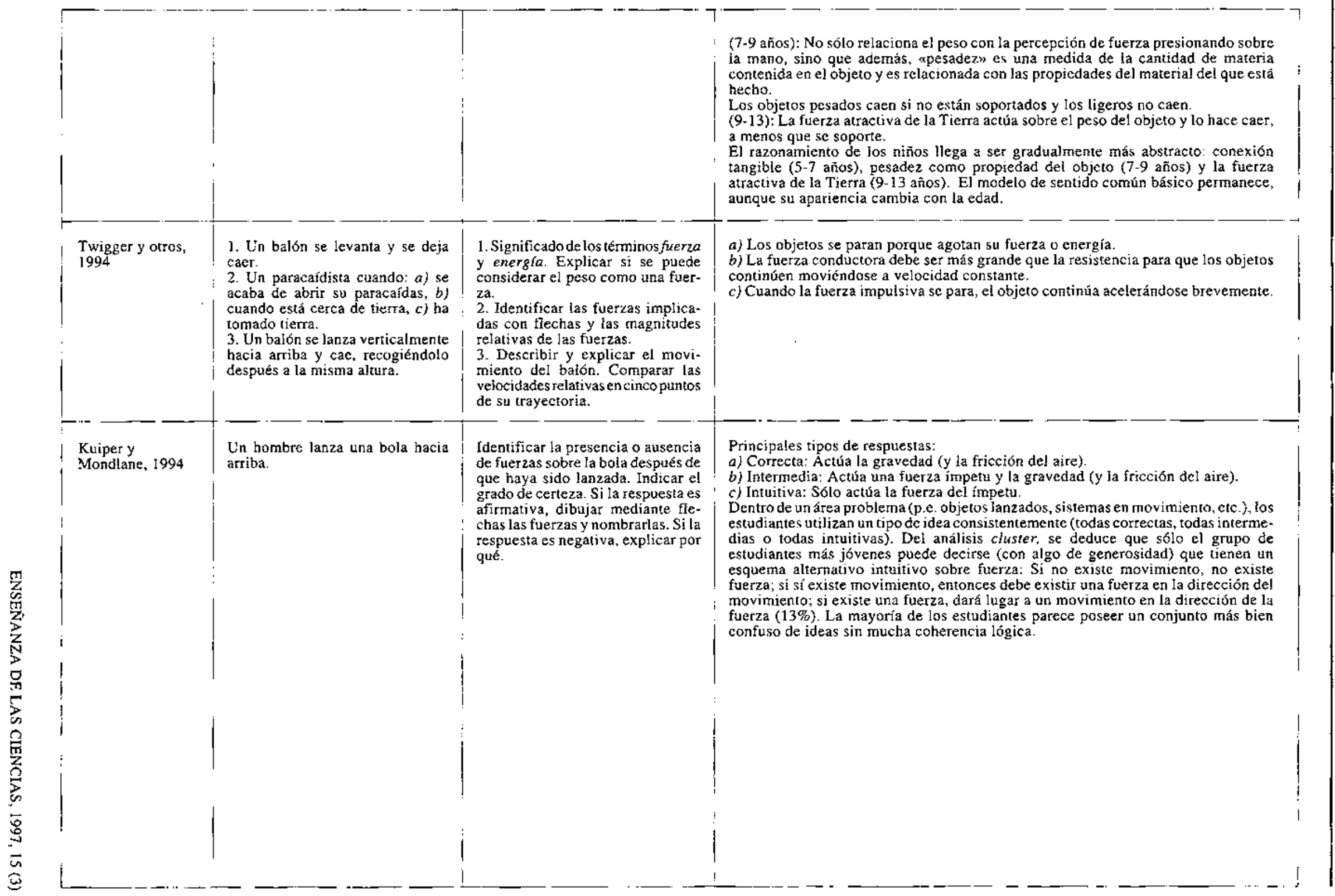


1. Probiemas de muelles: Se cuelga un peso de un muelle y se marca la longitud del mismo. Se quita el peso longitud. Se repite la operación con

dos pesos.
Este procedimiento fue usado orra Este procedimiento fue usado otra ver, cuando el mamero de pesos colgados del muelie la segunda vez fue tres, y después cuatro.

Algunas otras combinaciones también balancines: Se cuel equilibrado de balancines. Se cuelga un peso de un brazo de una palanca y se equilibra palanca se quita los pesos au palanca. Se quitan los pesos que con

1. Una monedas lanzada verticalmente hacia arriba vuelve a caer verticalmente 2. Un balon de baloncesto sigue una trayectoria parabolica hasta llegar a del aire.

3. La Luna en su trayectoria circular alrededor de la Tierra.

4. Un péndulo simple oscila de derecha a izquierda. Despreciar el rozamiento

5. Un péndulo compuesto gira (despreciar el rozamiento del aire). 6. Una piedri atada a una cuerda gira perpendicularmente al suelo. fueron usadas: dos y después tres pesos, dos y despues cuatro pesos. dos veces mediantediferentes conjuntos de pesos colgados en dos puntos con eldedoen los mismos dos puntos.

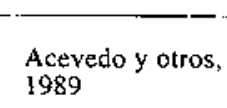
despreciando el rozamiento del aire.

\section{Actividad a realizar por el alumno}

Explicar qué hace que las cosas caigan al suelo. 2. Predecir la relación entre los tiempos que tardan al caer y explicarlo. mismo tiempo el suelo. 1

1. Identificar qué fuerza era mayor y expresar la relación entre las intensidades de esas fuerzas numericamente.

en esos dos puntensidad de las fuerzas de presión en esos dos puntos y expresar la relación entre ellas numérica.

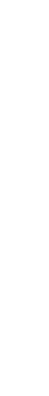

1. Identificar las fuerzas que actúan sobre la moneda en tres puntos de su trayectoria: cuando asciende, en la parre superior y cuando desciende. Dar una explicación.

tres puntos de fuerzas que actúan sobre el balon en parte más ale cumdectesciende. Daruscende, en la 3. Identificar las fuetzas que actúan sobre la Luna. Dar una explicacion.

4. Identificar las fuerzas que actúan sobre la bolita del péndulo en tres posiciones: en un punto cuando asciende, en la parte inferior y en un punto cuando desciende. Dar una explicación.

5. Identificar las fuetzas que actúan sobre la bolita. 6. Identuficar las fuerzas que actúan sobre la piedra en cuatro puntos de su trayectoria: arriba, abajo, en la posicion de las 9 horas cuando asciende y en la de las 2 cuando desciende. Dar una explicación.

\section{Resultados encontrados}

Nivel preoperacional: Concepciones basadas en sucesos correlacionados (causa efecto) evidentes. ( $« S_{1}$, porque tú las dejas caer,$n$ )

Nivei concreto inicial: Concepciones basadas en fuerzas no visibles unilaterales sobre un objeto único. Ejemplo de respuesta: porque algo tira de ellas hacia la Tierra.

Existe una cierta correspondencia entre el dominio del razonamiento lógico-matemático y el de la comprensión de conceptos en ciencias. Sin embargo, avances en un dominio tho son necesariamente dependientes del

El criterio que se utiliza para categorizar los datos son las etapas del pensamiento proporcional definidas por Karplus y Peterson (1970).

1. a) Respuestas cualitativas (p.e.: «la fuerza que se ejercía sobre el muelle es más alta la segunda vez porque se cuelgan más pesos). No podian responder a la segunda cuestion.

bespuesta ol problema un esuema aditivo. (p.e. «dos veces más» como respesta al probes us de un peso comparado con tres pesos.

c) Respuestas de tipo aditivo-muluplicativo. Solo usan un esquema multidi) Respuestas de relación 2:

e) Respuestas especiales (pe icativo. Usan razonamiento proporcional. émeros de los pesos para dar. 1:2 quiere decir tres veces, sumo los 2. a) Respuestas cualitativas.

b) Respuestas de esquema aditivo (contaban el número de agujeros o el numero de pesos y los sumaban o los restaban para dar la respuesta).

c) Respuestas de tipo multiplicativo-aditivo.

d) Respuestas de tipo multiplicativo.

e) Respuestas especiales.

Los niños de escuela elemental no identifican peso y fuerza nero son capaces dc usar la relación entre el número de pesos para comparar fuerzas. A partir de los 10 años, la mitad de los participantes eran cilpaces de dar la independiente del número de pes porta enteros.

Los estudiantes con competencia formal o concreta en el razonamiento proporcional dan respuestas alternativas a las del esquema newroniano gue injcialmente son similares.

2. Aparece una diferencia significativa entre esos individuos después del periodo de aprendizaje a favor de los alumnos con competencia formal. Los alumnos concretos no parecen progresar.

- Conclusiones.

La competencia formal en el razonamiento proporcional resulta condición necesaria para adquirit esquemas conceptuales como el newtoniano, pero no suficiente.

Justificación de los resultados: quizá el método de enseñanza no era todo lo bueno que podría esperarse, o bien el periodo de aprendizaje fue demasiado corto, o ambas cosas a la vez

Es frecuente la asociacion

- En los movimientos curvilíneos, existe una tendencia a introdacir fuerzas compensadoras de carácter centrifugo. 
estén mediatizadas por la propia pregunta; así, el alumno tiene que generar una respuesta sin que se le dé opción a reconsiderar el hecho físico presentado desde una perspectiva diferente a la que se deduce del marco teórico actual de lá mecánica clásica. Esto podría justificar el que los autores sólo describan las diferentes respuestas de los alumnos en términos de conceptos erróncos o ideas falsas (Helm, 1980), repuestas erróneas o correctas (Terry y Jones, 1986), nivel de comprensión adecuado o inadecuado (Gunstone y White, 1981), concepciones erróneas (Ivowi, 1984), errores de comprensión, de interpretación u omisiones (Galili, 1993). Salvo Gunstone y White (1981), que utilizan la predicción y la observación de un hecho físico concreto, el resto no puede decirse que presente cambios importantes en la metodología de investigación sobre la búsqueda de «lo que el alumno sabe» acerca del concepto de fuerza. Además, todos los autores, sin excepción, realizan las descripciones de las respuestas de Jos alumnos a partir del marco teórico que se deduce de la mecánica clásica; es decir, desde el propio contenido objeto de búsqueda, hecho encontrado $y$ descrito por otros autores (Hashwesh, 1988; Abimbola, 1988; Marín, 1994; entre otros).

\section{Comparaciones entre los resultados obtenidos en Jos estudios categorizados como descriptivos inde- pendientes, referidos a las situaciones de lanzamiento o caída de objetos (Tabla IV)}

Para hacer referencia a las interpretaciones que hacen los investigadores o educadorcs a las respuestas de los alumnos en esta categoría se van a utilizar los términos esquemas alternativos (propuestos por Watts, 1983 , para referirse a las diferentes explicaciones que dan los alumnos, independientes de los hechos físicos presentados, y alternativos a los que poseen los miembros de la comunidad científica actual) que tienen los alumnos sobre el concepto de fuerza.

Para observar si se ha producido progresión en la delimitación de los esquemas alternativos (EA), se comparan los trabajos publicados en el periodo 1979-85 con los de 1985-95, referidos al concepto de fuerza:

\section{Esquemas a subesquemas alternativos deducidos en el periodo 1979.85}

EA1: La fuerza es considerada como una propiedad ligada al cuerpo (Viennot, 1979). De este esquema podrian derivar los subesquemas siguientes:

- Los humanos, las máquinas y algunos objetos son centros de fuerza: noción antropomórfica de fuerza (Clement, 1973; Watts, 1983).

- Las fuerzas son obligaciones para realizar una acción cn contra de alguna resistencia (Watts, 1983).

- La fuerza es una acción que realiza alguien o algo: la cantidad de fuerza es proporcional a la cantidad de actividad (Watts, 1983).
- Los objetos retenidos en una posición tienen fuerza (Watts, 1983).

EA2: Todo cuerpo en movimiento lleva asociado una fuerza (Viennot, 1979). De cste esquema se derivan los subesquemas siguientes:

- Las fuerzas son necesarias para mantener un cucrpo en movimiento (Viennot, 1979).

- Si un cuerpo está moviéndose, existe una fuerza neta sobre él en la dirección del movimiento (Viennot, 1979)

- La fuerza es proporcional a la velocidad (si $V=0 \Rightarrow$ $F=0$, incluso si $a \neq 0$; si $V \neq 0 \Rightarrow F=0$, incluso si $a=$ 0 ; si las velocidades son diferentes, las fuerzas son distintas, incluso si las aceleraciones son iguales) (Viennot, 1979).

- Cuando dos fuerzas se juntan, pueden combinarse para cambiar el movimiento de los objetos (Watts, 1983).

EA3: El cambio de velocidad es consecuencia de la fuerza (Viennot, 1979)

Esquemas o subesquemas alternativos deducidos' en la década 1985-95:

Se ha encontrado el siguiente referido a situaciones físicas de lanzamiento o caida de objetos:

- Las fuerzas de rozamiento tienen un valor constante (Thijs, 1992).

De la comparación del catálogo de esquemas alternativos entre los dos periodos (1979-85 y 1985-95), se observa que la mayoría de eltos se hicieron explícitos en el primer periodo. En el segundo, se han ratificado y matizado, tal como se observa en los trabajos de Bohea (1990), Finegold y Gorsky (1991), Thijs (1992) y Kuiper y Mondlane (1994), los cuales utilizan semejantes hechos físicos que Viennot (1979) y Watts (1982 y I983), $\mathrm{y}$, por tanto, obtienen análogos resultados:

a) Una crítica al esquema $E A .2$, por no ser un buen predictor para las situaciones físicas que se refieren a movimientos periódicos, lineales o tiro de proyectiles (Finegold y Gorsky, 1991).

b) Estudios evolutivos, trabajos en tos que se pone de manifiesto la evolución con la edad de los esquemas o subesquemas anteriores, que se han publicado en la década de 1985-95 (Reynoso et al., 1993; Bar et al., 1994).

c) Matizan Ios EA.2 en el sentido de que sólo los alumnos más jóvenes presentan dicho esquema alternativo y también detectan que los estudiantes de mayor edad parecen poseer un conjunto de ideas sin mucha coherencia logica (Kuiper y Mondlane, 1994).

Para el caso de lanzamiento o caída de objetos, los esquemas y subesquemas que se obtienen para la grave- 
dad y conceptos con ella relacionados, en el periodo 1979-85, son Ios propuestos por Watts (1982) y Ruggiero y otros (1985, Tabla IV).

En el periodo 1985-95, los resultados que aparecen referidos al concepto de gravedad y conceptos con ella relacionados, van encaminados a matizar los esquemas o subesquemas of recidos por Watts (1982) y Ruggiero y otros (1985). Así, por ejemplo:

a) Noce y otros (1988) observan que los esquemas y subesquemas propuestos por Ruggiero y otros (1985) no se dan en sujetos con edades inferiores (8-12 años). Al utilizar muestras diferentes, observan la existencia de un modelo descriptivo y otro explicativo que utilizan los niños en sus respuestas acerca de la caída de objetos. De esta forma matizan, por ejemplo, el modelo explicativo geocéntrico propuesto por Ruiggero y otros (1985), que relaciona la fuerza de la gravedad con la Tierra, y proponen el esquema: La gravedad es la fuerza que hace que los objetos floten (definición tautológica).

b) Reynoso y otros (1993) realizan un estudio evolutivo y pueden detectar en qué edad aparecen los diferentes esquemas altenativos. Así, por ejemplo, encuentra que, entre los profesores de primaria, el esquema mas frecuente es que digan que «la fuerza de la gravedad en la Tierra es mayor que en la Luna o que la fuerza de la gravedad en la Luna es cero».

c) Bar y otros (1994) realizan también un estudio evolutivo con una muestra de sujetos entre Ios 4 y 13 años, lo que les permite asociar las diferentes formas de explicar los hechos físicos presentados con la edad (Tabla IV).

De la comparación de los trabajos publicados en el primer periodo (1979-85) con los del segundo, referidos al concepto de gravedad y conceptos con ella relacionados, se observa que: a) la casi totalidad de esquemas y subesquemas aparece en los trabajos publicados en el primer periodo; $y, b$ ) los del segundo periodo progresan hacia estudios de carácter evolutivo, lo que implica un cambio metodológico y de objetivos en la investigación, al menos en lo que se refiere a la selección de la muestra.

Como se pone de manifiesto en el análisis de los $E A$ para fuerza, gravedad y conceptos con ella relacionados, no parece deducirse que exista progresión en los resultados, así:

a)Los trabajos realizados con posterioridad a 1985 no parecen aportar datos novedosos en cuanto a posibles esquemas alternativos que tienen los alumnos con utilidad para la enseñanza. Incluso se podría afirmar que los trabajos anteriores a 1985 ofrecen resultados más extensos y de mayor argumentación que los realizados con posterioridad, a excepción de los hechos físicos, las cuestiones y muestras diferentes utilizadas.

b)EI carácter empírico de los diferentes trabajos, si se comparan las dos décađas, es también análogo. c) En la tabla IV se observa que los autores utilizan diferentes términos para referirse a análogos resultados. Así, por ejemplo, para referirse a que «el movimiento lieva asociado una fuerza», Viennot (1979) le llama noción de fuente de fuerza (supply of force); Watts (1983) y Boeha (1990), fuerza móvil (motive forces); y Twigger y otros (1994), fuerza conductora (driving force). Todo ello indica que, si se compara la terminología utilizida en los trabajos realizados en el periodo 1979-85 con los del periodo $1985-95$, no se ha producido, en muchos casos, ni tan siquiera unanimidad en los términos a utilizar y menos aún en su significado (Jiménez Gómez, Solano y Marín, 1994). Y si esto es así, obviamente, cabe esperar que exista aún menor consenso en la metodología de investigación, así como en el contexto teórico que marque líneas de actuación en las búsquedas de «lo que el alumno sabe».

Se observa, desde el punto de vista metodológico, una tendencia entre los trabajos publicados en el segundo periodo, y para esta categoría, que se refiere al aumento de estudios evolutivos y los cambios que de ello se derivan.

\section{Comparaciones entre los resultados obtenidos en los estudios categorizados como descriptivos relaciona- les (TabIa V)}

No es frecuente realizar trabajos donde se relacionen las respuestas de los alumnos con sus manifestaciones cognoscitivas: y que han mostrado su relevancia en otros trabajos (rivel cognoscitivo, estilo cognitivo, nivel de inteligencia, etc.), como se pone de manifiesto en la reducida muestra que integra esta categoría. No obstante, se quiere indicar que, si en la selección bibliográfica hubiesen entrado revistas del ámbito de la psicología o sobre otro contenido objeto de enseñanza diferente, quizás los resultados hubiesen sido distintos, pero nosotros hemos contextualizado este trabajo en el ámbito de las revistas especialidas en el área de didáctica de las ciencias y para el concepto de fuerza.

La importancia de realizar estos estudios relacionales reside en la posibilidad de contrastar la coherencia y validez de ła información obtenida del alumno con otros datos que sí parecen estar bien contrastados, así como analizar e interpretar las «ideas» de los alumnos de un modo más amplio y contextualizado, dado que la mejor descripción de la cognición del alumno no debe limitarse a dichas «ideas» (Marín, 1995). Sin embargo, ninguno de los trabajos analizados (en esto no se distinguen los trabajos del primer periodo de los del segundo) consigue estos objetjvos, pues se limitan a establecer relaciones entre datos y a cruzarlos estadísticamente.

La ausencia del entramado teórico donde están implicados los factores cognoscitivos utilizados en los diferentes trabajos limita notablemente las conclusiones propuestas, quedando reducidas las posibilidades que ofrecen los trabajos enmarcados dentro de esta categoría. Así, las diferencias entre los trabajos de Selman y otros 
(1982) y de Bar (1989), aun utilizando muestras de sujetos parecidas, los diferentes resultados que ofrecen, pensamos que se deben al tipo de categorización utilizada y las diferencias de Ios hechos físicos presentados.

\section{CONCLUSIONES}

De las comparaciones realizadas anteriormente se pueden extraer las siguientes conclusiones:

1. Si se aplica el criterio de Lakatos (se determina si tuna teoría es progresiva a través de su capacidad de predecir hechos nuevos o, lo que es lo mismo, si su desarrollo teórico anticipa su desarrollo empírico [1974, 1983]) a los estudios realizados sobre "lo que el alumno sabe» sobre fuerza, se puede observar que el movimiento de las concepciones alternativas no presenta indicios de progresión, al menos no se evidencia a través de los resultados ofrecidos por los diferentes trabajos seleccionados sobre fuerza, pues todos los trabajos, independientemente del periodo de publicación (1979-85 o 1985-95), presentan un carácter fundamentalmente empírico y no se aprecia que el desarrollo teórico se anticipe a los resultados.

2. En algunos aspectos, se aprecia cierto progreso durante la ülima década (1985-95) en la identificación y descripción de las respuestas de los alumnos acerca de las nuevas preguntas y situaciones físicas presentadas, pero no en cuanto a:

a) el número de esquemas alternativos que presentan los sujetos y que aparecen descritos en los trabajos publicados con anterioridad a 1985 ;

b) el carácter global con el que los sujetos interpretan los hechos físicos. Así, Viennot (1979) y Watts (1983) describen que los sujetos globalizan el concepto de fuerza con el de energía y cantidad de movimiento, pero este hecho posteriormente no se trabaja ni se intenta estudiar la posible psicogénesis del concepto de fuerza (como lo hace, por ejemplo, Piaget et al., 1973).

En sentido estricto, el criterio de Lakatos, tal y como se estableció, podría ser adecuado para evaluar el grado de progresión de las teorías científicas, por lo que, contrarios al trabajo de Gilbert y Swift, no debería aplicarse sobre el MCA, dado que esta corriente didáctica ni siquiera posee un entramado teórico que sirva como substrato de las aportaciones empíricas, pues los trabajos sobre detección de «lo que el alumno sabe» sobre fuerza y conceptos con ella relacionados, que en el periodo inicial son marcadamente descriptivos y realizan la búsqueda de información exclusivamente determinada por el propio contenido objeto de búsqueda, continúan manteniendo estas características en el segundo periodo analizado (1985-95).

Podría mantenerse que el entramado teórico para delimitar las ideas previas del alumno lo constituyen los pro- pios contenidos académicos pero, si esto es así, a la vista de los resuitados obtenidos, todo parece indicar que los estudios de «lo que el alumno sabe» sobre fuerza es una línea de investigación que parece concluida, en la medida que no se pueden realizar nuevas aportaciones novedosas o que profundicen más en la cognición del alumno, por lo que habría que concluir que el entramado teórico utilizado se podría considerar inadecuado.

Esta sensación de techo hace pensar en la imposibilidad de continuidad, futuro y perspectiva. Sin embargo, pensamos que los trabajos que aquí se han seleccionado y analizado sólo constituyen una primera aproximación al problema de delimitar «lo que el alumno sabe», que han permitido al profesor cubrir, entre otros, los siguientes objetivos (Marín y Jiménez Gómez, 1992):

- Mejorar la comunicación entre los profesores y alumnos durante el desarrollo del acto didáctico (Driver, 1989).

- Realizar nuevos diseños de enseñanza (Osborne y Freyberg, 1985; Posner et al., 1982; Nussbaum y Novick,1981, entre otros).

- Generar una nueva perspectiva sobre el aprendizaje (Driver y OIdhan, 1985; Glaser y Bassok, 1989, entre otros).

\section{- Ayudar a conocer mejor los procesos de aprendizaje.}

- Servir de guia para la investigación educativa, incluso si se trabaja en un contexto externo a los procesos cognitivos.

- Ayudar al profesor a interpretar los sucesos que tienen lugar en el aula, especialmente en la toma de decisiones.

A pesar de estas mejoras, es posible progresar si se hace una revisión de cuál es la información que se puede obtener del alumno de interés didáctico y, a la vez, se utilizan contextos teóricos que permitan un mayor acercamiento a la realidad cognoscitiva del alumno, lo que llevaría necesariamente a un cambio metodológico en la búsqueda de esta información.

Al fin y al cabo, no se puede negar que la búsqueda de «lo que el alumno sabes se hace con el objetivo primordial de acomodar los diseños de enseñanza a sus peculiaridades cognoscitivas $y$, en tanto se obtenga una información más cercana a estas peculiaridades, los diseños de enseñanza quedarán más acomodados y consecuentemente se incrementará la eficacia didáctica.

Se podría entonces pensar que la información que aportan los diferentes trabajos seleccionados sobre cl alumno es suficiente para realizar diseños de enseñanza, pero pensamos que habría que dudar de la eficacia didáctica de estos diseños, puesto que los resultados a los que llegan están excesivamente mediatizados por la lógica del contenido académico objeto de búsqueda, pues no se deben olvidar las importantes diferencias que suelen existir entre el contenido académico y el del alumno (Marín y Jiménez, Gómez, 1992). 
Creemos que habría que abordar los estudios sobre «lo que el alumno sabe» desde una perspectiva más amplia, que permitiera determinar al educador:

a) los momentos claves (edad, por ejemplo) en los cuales los alumnos pasan de la globalización del concepto de fuerza a su diferenciación, por lo que sería necesario realizar estudios de carácter evolutivo, relativos a dicho concepto;

b) las limitaciones y capacidades cognitivas de los sujetos parat aprender un determinado concepto, en este caso el de fuerza y conceptos con ella relacionados;

c) tratar de ajustar las actividades de enseñanza a las características cognitivas, motivacionales y afectivas del alumno; hecho que sólo es posible realizar si se conoce del sujeto toda aquella información significativa del alumno de interés didáctico, tanto si está relacionada con el contenido académico como si no lo está (Marín, 1984).

Llegar a solucionar los puntos anteriores requiere, previamente, que los educadores e investigadores clarifiquen cuestiones elementales y útiles de cara a conseguir una progresión en este tipo de estudios (¿qué entiende cada educador o investigador por «lo que el alumno

\section{REFERENCIAS BIBLIOGRÁFICAS}

ABIMBOLA, I.O. (1988). The problem of terminology in the study of student conceptions in Science. Science Education, Vol. 72(2), pp. 175-184.

ACEVEDO, J.A., BOLÍVAR, J.P., LOPEZ MOLINA, E.I. y TRUIILLO, M. (1989). Sobre las concepciones en dinámica elemental de los adolescentes formales y concretos y el cambio metodológico, Enseñanza de las Ciencias, Vol. 7(1), pp. 27-34.

BAR, V. (1989). Introducing mechanics at the elementary school. Physics Education, Vol. 24, pp. 348-352.

BAR, V, ZINN, B., GOLDMUNTZ, R. y SNEIDER, C. (1994). Children's concepts about weight and free fall. Science Education, Vol. 78(2), pp. 149-169.

BOEHA, B.B. (1990). Aristotle, alive and well in Papua New Guinea science classrooms. Physics Education, Vol. 25, pp. 280-283.

BROWN, D.E. (1989). Students' concept of force: the importance of understanding Newton's third law. Physics Education, Vol. 24, pp. $352 \cdot 358$.

BUNGE, M. (1981). Lainvestigación científica Ariel: Barcelona.

CARMICHAEL, P. et al. (1990). Rechearh on students' conceptions in science: a bibliography. Children's learning in Science: University of Leeds.

CLEMENT, J., BROWN, D.E. y ZIETSMAN, A. (1989). Not all preconceptions are misconceptions: finding «anchoting conceptions» for grounding instruction on students' intuitions. International Journal of Science Education, Vol. 11, núm. especial, pp. 554-565. sabe» sobre un determinado contenido đe enseñanza?); ¿qué marco teórico es el más adecuado para realizar este tipo de tratıajos?; ¿qué metodología es la más adecuada?; etc.), en vista de lo cual, no parece que la afirmación «el catálogo de concepciones sea bastante completo por lo que habría que dedicar los esfuerzos en otras direcciones» sea ac ərtada.

Para finalizar, habría que decir que es necesario realizar un estudio profundo acerca de las metodologías y marcos teóricos que subyacen en los trabajos seleccionados, y que han constituido la base de nuestro análisis, para, a partir de aquí, intentar hacer confluir lo que otros educadores e investigadores han reclamado (López Rupérez, 1990), la nıcesidad de realizar una reflexión teórica, respecto a los paradigmas educativos que han surgido de la filosofía de la ciencia en la tradición del cambio conceptual y de la psicología genética, dando lugar a un nuevo programa de investigación más fructífero y mejor fundamentado teóricamente (Marín, 1994).

\section{NOTA}

El presente trabajo ha sido financiado por la DGICXT como parte del proyesto PS93-0174 del Programa Sectoralde Promoción General del Conocimiento.

CONFREY, J. (1990). A Review of the Research on Student Conceptions in Mathematics, Science and Programming, pp. 3-56, en C. Cazden (eds.). American Education Research Association. Michigan State University. Review of Educational Research.

DRIVER, R. y OLDHAM, V. (1985). Constructivist Approuch to Curriculum Development in Science. Paper prepared for the Symposium. Personal Construction of Meaning in Educational Settings. Bera, Sheffield.

DRIVER, R. (.989). Studicnts'conceptions and the learning of science. International Journal of Science Education, Vol. 11, núm. especial, pp. 481-490.

FINEGOLD, M. y GORSKY, P. (1991). Students' concepts of force as applied to related physical systems: A search for consistency, International Journal of Science Education, Vol. 13(1), pp. $97-113$.

GALILI, I. (1993). Weight and gravity: teachers' ambiguity and student; confusion about the concepts. International Journal of S'cience Education. Vol. 15(2), pp. 149-162.

GALILI, I. y BAR, V. (1992). Motion implies force: where to expect vestiges of the misconception? International Journal of Science Education, Vol. 14(1), pp. 63-81.

GILBERT, J.K. y SWIFT, J. (1985). Towards a Lakatosian analysis of the Piagetian and alternative conceptions research programs. Science Education, Vol. $69(5)$, pp. 681-696.

GLASER, R. y BASSOK, M. (1989). Learning Theory and the Study of Instruction. Ann. Rev. Psychol, 40. pp. $631-666$. 
GÓMEZ, M.A., POZO, J.I., SANZ, A. y LIMÓN, M. (1992) La estructura de los conocimientos previos en química: una propuesté de núcleos conceptuales. Investigación en la Escuela, 18 , pp. $23-40$

GUNSTONE, R.F. y WHITE, R.T. (1981). Understanding of gravity. Science Education, Vol. 65(3), pp. 291-299.

GUNSTONE, R.F. y WHITE, R.T. (1981). Understanding of gravity. Science Education, Vol. 65(3), pp. 291-299.

HASHWFH, M.Z. (1988). Descriptives studies of students' conceptions in science, Journal of Research in Science Teaching, Vol. 25(2), pp. 121-134.

HELM, H. (1980). Misconceptions in physics amongst South African students. Physiscs Eduaction, Vol. 15, pp. 92-105.

IVOWI, U.M.O. (1984). Misconceptions in physics amongst Nigerian secondary school students. Physics Education, Vol. 19, pp. 279-285

JIMÉNEZ GÓMEZ, E., SOLANO, I. y MARÍN, N. (1994). Problemas de terminología en estudios realizados sobre «lo que el alumno sabe» en ciencias. Enseñanza de las Ciencias, Vol. 12(2), pp. 235-245.

KUHN, T.S.(1981). La estructura de las revolucionescientíficas. Madrid: Fondo de Cultura Económica

KRUGER, C., PAI,ACIO, D. y SUMMERS, M. (1992). Surveys of English Primary Teachers' Conceptions of Force, Energy and Materials. Science Education, Vol. 76(4), pp. 339-35l.

KUIPER, I. y MONDLANE, E. (1994). Student ideas of science concepts: alternative frameworks? Intemational Journal of Science Education, Vol. 16(3), pp. 279-292.

LAKATOS, I. (1974). Historia de la cienciay sus reconstrucciones racionales. Madrid: Technos.

LAKATOS, I. (1983). La metodología de los programas de investigación científica. Madrid: Alianza Universitaria.

LOPEZ RUPÉREZ, F. (1990). Epistemologia y didáctica de las ciencias. Un análisis de segundo orden. Enseñanza de las Ciencies, Vol. 8(1), pp. 65-74.

MALONEY, D.P. (1984). Rule-governed approaches to physicsNewton's third law. Physics Education, Vol. 19, pp. 37-42.

MARÍN, N. (1994). Tesis doctoral. Universidad de Granada.

MARÍN, N. (1995). Metodología para obtener información del alumne de interés didáctico. Servicio de Publicaciones de Almeria. Universidad de Almería.

MARÍN, N. y BENARROCH, A. (1994). A comparative study of Piagetian and constructivist work on conceptions in science. International Journal of Science Education, Vol. 16(1), pp. 1-15.

MARÍN, N. y JIMÉNEZ GÓMEZ, E. (1992). Problemas metodológicos en el tratamiento de las concepciones de los alumnos en el contexto de la filosofía e historia de la ciencia. Enseñanza de las Ciencias, Vol. 10(3), pp. 335-339.

MONTANERO, M. y PÉREZ, A.L., (1995). A survey of Students' understanding of colliding bodies. Physics Education, Vol. $30(5)$, pp. $277-283$

NOCE, $G .$, TOROSANTUCCI, G. Y VICENTINI, M. (1988). The floating of objects on the moon: Prediction from a theory or experimental facts? International Journal of Science Education, Vol. 10(1), pp. 61-70.
NUSSBAUM, J. y NOVICK, S. (1981). Brainstorming in the classroom to invent a model: a case study. School Science Review, 221, p. 62.

OSBORNE, R. y FRYBERG, P. (1985). Learning in Science. The implication of children's science. Londres: Heineman.

PIAGET, J. et al. (1973). La formation de la notion de force. París: PUF.

POSNER et al. (1982). Accomodation of a Scientific Conception: Toward a Theory of Conceptual Change. Science Education, Vol. 66, pp. $21 \mathrm{l}-227$.

REYNOSO, H.E., ENRIQUE FIERRO, H., GERRDOTORRFS, O., VICENTINI-MISSONI, M. y PEREZ DE CEL,IS, J.H. (1993). The alternative frameworks presented by Mexican students and teachers concerning the free fall of bodies. International Journal of Science Education, Vol. 15(2), pp. 127-138.

RUGGIERO, S., CARTELLI, A., DUPRE, F. y VICENTINIMISSONI, M. (1985). Weight, gravity and air pressure: Mental representations by Italian middle school pupils. European Journal of Science Education, Vol. 17(2), pp. $181-194$.

SELMAN, R.L., KRUPA, M.P., STONE, C.R. y JAQUETIE, D.S. (1982). Concrete operational thought and the emergence of the concept of useen force in children's theories of electromagnetism and gravity. Science Education, Vol. $66(2)$, pp. 181-194.

TERRY, C. y JONES, G. (1986). Alternative frameworks: Newton's third law and conceptual change. Europeun Journal of Science Education, Vol. 8(3), pp. 291-298.

TERRY, C., JONES, G. y HURFORD, W. (1985). Children's conceptual understanding of forces and equilibrium. Physics Education, 20, pp. 162-165.

THIIS, G.D. (1992). Evaluation of an Introductory Course on «Force» considering students" preconceptions. Science Education, 76(2), pp. 155-174

TWIGGER, D., BYARD, M., DRIVER, R., DRAPER, S. et al. (1994). The conception of force and motion of students aged between 10 and is years: an interview study designed to guide instruction. International Journal of Science Education, Vol. 16(2), pp. 215-229.

VIENNOT, L. (1979). Spontaneous reasoning in elementary dynamics. European Journal of Science Education, Vol. 1(2), pp. 205-221.

VILLANI, A. y PACCA, J. (1990). Conceptos espontáneos sobre colisiones. Enseñanza de las Ciencias, Vol. 8(3), pp. 238-243.

WATTS, D.M. (1982). Gravity-don't take it for granted. Physics Education, Vol. 17, pp. 116-121.

WATTS, D.M. (1983). A study of schooichildren's alternalive frameworks of the concept of force. European Journal of Science Education, Vol. 5(2), pp. 217-230.

WATTS, D.M. y ZYLBERSZTA N, A. (1981). A survey of some children's ideas about force. Physics Education, Vol. $16(6)$, pp. $360-365$

WATTS, D.M. (1983). A study of schoolchildren's alternative frameworks of the concept of force. European Journal of Science Education, Vol. 5(2), pp. 217-230.

[Artículo recibido en abril de 1996 y aceptado en abril de 1997.] 